\title{
UNIQUENESS OF EQUILIBRIUM CONFIGURATIONS IN SOLID CRYSTALS*
}

\author{
WILFRID GANGBO ${ }^{\dagger}$ AND ROBERTO VAN DER PUTTEN PU $^{\ddagger}$
}

\begin{abstract}
In this article, under suitable assumptions, it is proved that $\inf _{\mathbf{u} \in \mathcal{U}_{\Lambda}} E[\mathbf{u}]$ is dual to $\sup _{(a, b)}\left\{\int_{\Omega} a(\mathbf{F}(\mathbf{x})) d \mathbf{x}+\int_{\Lambda} b(\mathbf{y}) d \mathbf{y}\right\}$, where, $E[\mathbf{u}]:=\int_{\Omega}(h(\operatorname{det} D \mathbf{u})-\mathbf{F} \cdot \mathbf{u}) d \mathbf{x}$. Here, the infimum is performed over $\mathcal{U}_{\Lambda}$, the set of all orientation-preserving deformations $\mathbf{u} \in C^{1}(\Omega)^{d}$ that are homeomorphisms from $\bar{\Omega}$ onto $\bar{\Lambda}$, and the supremum is performed over the set of all upper semicontinuous functions $a, b$ such that $a(\mathbf{z})+\alpha b(\mathbf{y}) \leq h(\alpha)-\mathbf{y} \cdot \mathbf{z}$. This duality result turns out to be important in the study of existence and uniqueness of smooth minimizers of $E$. Note that $M \rightarrow h(\operatorname{det} M)$ is not coercive and thus direct methods of the calculus of variations don't apply here.
\end{abstract}

Key words. Monge-Kantorovich, rearrangement, duality, solid crystals

AMS subject classifications. 49J40, 28A50

PII. S0036141099356684

Introduction. The theory of duality, one of the main tools in the calculus of variations, is well developed within the context of convex variational problems of the form $\inf _{\mathcal{U}} \int_{\Omega} L(\mathbf{x}, \mathbf{u}(\mathbf{x}), D \mathbf{u}(\mathbf{x})) d \mathbf{x}$, where the real-valued function $M \rightarrow L(\mathbf{x}, \mathbf{u}, M)$ defined on the set $\mathbf{R}^{d \times d}$ of the $d \times d$ matrices is convex for each $\mathbf{x} \in \Omega$ and $\mathbf{u} \in \mathbf{R}^{d}$. We recall that in the particular case $L(\mathbf{x}, \mathbf{u}, M)=g(M)-\mathbf{F}(\mathbf{x}) \cdot \mathbf{u}$, where $g$ is convex and coercive, then the duality statement is as follows: the infimum

$$
\inf \left\{\int_{\Omega} L(\mathbf{x}, \mathbf{u}(\mathbf{x}), D \mathbf{u}(\mathbf{x})) d \mathbf{x}: \mathbf{u} \in W_{0}^{1, p}\left(\Omega, \mathbf{R}^{d}\right)\right\}
$$

and the supremum

$$
\sup \left\{-\int_{\Omega} g^{*}(-\mathbf{p}(\mathbf{x})) d \mathbf{x}: \mathbf{p} \in L^{q}\left(\Omega, \mathbf{R}^{d \times d}\right), \operatorname{div} \mathbf{p}=\mathbf{F}\right\}
$$

coincide, where $g^{*}$ is the Legendre transform of $g$. Furthermore, the extremum is attained in both problems (see [10]). An important class of nonconvex functions that occur in nonlinear elasticity theory is the class of polyconvex functions. There is no available theory of duality for that class. Recall that a real-valued function $W$ of $\mathbf{R}^{d \times d}$ into $\mathbf{R} \cup\{+\infty\}$ is said to be polyconvex if it can be written as a convex function of the minors of $M$ (see [8]). In this paper we consider a special class of polyconvex functions of the form $L(\mathbf{x}, \mathbf{u}, M):=W(M)-\mathbf{F}(\mathbf{x}) \cdot \mathbf{u}$ and introduce a maximization problem, dual to $\inf _{\mathcal{U}} \int_{\Omega} L(\mathbf{x}, \mathbf{u}, D \mathbf{u}) d \mathbf{x}$. As an application we study stable configurations of solid crystals occupying a reference configuration $\Omega$ and subject to a body force $\mathbf{F}$. If the crystal undergoes a deformation represented by a map $\mathbf{u}: \Omega \rightarrow \mathbf{R}^{d}, d \geq 2$ (in general $d=3$ ), then its total energy functional is

$$
E[\mathbf{u}]:=\int_{\Omega}(W(D \mathbf{u})-\mathbf{F} \cdot \mathbf{u}) d \mathbf{x},
$$

*Received by the editors May 24, 1999; accepted for publication (in revised form) January 13, 2000; published electronically September 15, 2000.

http://www.siam.org/journals/sima/32-3/35668.html

†School of Mathematics, Georgia Institute of Technology, Atlanta, GA 30332 (gangbo@math. gatech.edu). This author was supported by NSF grants DMS-96-22734 and DMS-99-70520.

${ }^{\ddagger}$ Dipartimento di Matematica, Universita di Genova, Genova 16146, Italy (vanderpu@dima.unige. it). 
where $W$ represents the Helmholtz free energy density. In the framework of the continuum theory proposed by Ericksen [11] and [12], which has stimulated a growing body of work (see [23], [22], [21], [20], [25], [29], [28], [27], [26]), $W$ belongs to a class of energy density functions that are invariant under change of lattice basis and frame:

$$
W(M)=W(Q M H)
$$

for all $M \in \mathbf{R}^{d \times d}$, all $Q \in \mathbf{R}^{d \times d}$ such that $Q^{T} Q=I$, $\operatorname{det} Q>0$, and all $H \in \mathbf{Z}^{d \times d}$, $|\operatorname{det} H|=1$. The class of the energy densities suggested by Ericksen contains those of the form

$$
W(M)=h(\operatorname{det} M) \quad\left(M \in \mathbf{R}^{d \times d}\right),
$$

where $h$ is a convex function. In fact, it was shown by Chipot and Kinderlehrer [7] and Fonseca [15] that if $W$ is of the form (1), then its quasi-convex envelope $Q W$ is of the form (2). Let us point out that the class of functions in (1) does not fall in the updated class of energy density functions of solid crystals. However, for purely mathematical interest, in what follows we choose to study the case where $W$ satisfies (1), $Q W=W$, and we still interpret the functional $E$ as a solid crystal energy functional.

Following previous works (see, for instance, [17]) we assume that

$$
h \in C^{2}(0,+\infty) \text { is strictly convex, }
$$

$$
h(t) \rightarrow+\infty \text { as } t \rightarrow 0^{+} \text {and } h(t) / t \rightarrow+\infty \text { as } t \rightarrow+\infty .
$$

We extend $h$ to $\mathbf{R}$ by setting

$$
h(t):=+\infty \text { if } t \leq 0 .
$$

Requirements (4) and (5) are imposed to make it energically impossible to compress part of the body of the crystal to zero volume, to extend part of the body excessively, or to change orientation. A typical example of body force is the gravity $\mathbf{F}=-g \mathbf{e}_{d}$, which can be written as the $L^{1}$-limit of a sequence of diffeomorphisms. Here we have set $\mathbf{e}_{d}:=(0, \ldots, 0,1)$.

If the crystal undergoes a deformation $\overline{\mathbf{u}}$ under the action of the body force $\mathbf{F}$, then

$$
-\operatorname{div}\left(\sigma_{\overline{\mathbf{u}}}\right)=\mathbf{F} \quad \text { in } \Omega
$$

where $\sigma_{\overline{\mathbf{u}}}$ is the stress tensor $\frac{\partial W}{\partial M}(D \overline{\mathbf{u}})$. Solutions of (6) could be interpreted as critical points of the functional $E$.

A problem of great interest in nonlinear elasticity is the so-called pure displacement boundary value problem: given a diffeomorphism $\mathbf{u}_{o}$ from $\bar{\Omega}$ onto $\bar{\Lambda}$, where $\Lambda \subset \mathbf{R}^{d}$ is an open, bounded set, find $\overline{\mathbf{u}}$ stable solution of (6) such that the restrictions of $\overline{\mathbf{u}}$ and $\mathbf{u}_{o}$ on $\partial \Omega$ coincide. Stability means that not only is $\overline{\mathbf{u}}$ a critical point of $E$, but $\overline{\mathbf{u}}$ minimizes $E$ over $\mathcal{U}_{o}$, the set of all maps $\mathbf{u}$ from $\bar{\Omega}$ onto $\bar{\Lambda}$ that are in $C^{1}(\Omega)^{d}$, $\operatorname{det} D \mathbf{u}>0$, and such that the restrictions of $\mathbf{u}$ and $\mathbf{u}_{o}$ on $\partial \Omega$ coincide. Since $M \rightarrow h(\operatorname{det} M)$ is not coercive, and $\mathcal{U}_{o}$ is not closed under the weak topology on $L^{p}$ spaces, the problem of minimizing $E$ over $\mathcal{U}_{o}$ escapes the classical methods of the calculus of variations, and there is currently a wide literature on the subject. When $\mathbf{u}_{o}$ is the identity map and $\mathbf{F}=-g \mathbf{e}_{d}$ is the gravity force, Fonseca and Tartar [17] 
showed that $E$ has infinitely many minimizers in the set of displacements that are in $W^{1, \infty}(\Omega)^{d}$. Also, Chipot and Kinderlehrer [7] proved for $E$ existence of parametrized measure minimizers by enlarging the set $\mathcal{U}_{o}$ to a set of Radon measures. We show that if $\mathbf{F} \in C^{1}(\bar{\Omega})^{d}$ is a homeomorphism, such that $\operatorname{det} D \mathbf{F} \in C^{1}(\bar{\Omega})^{d}, \operatorname{det} D \mathbf{F}>0$, if $\Lambda$ and $\mathbf{F}(\Omega)$ are convex, then the infimum

$$
\inf _{\mathcal{U}_{o}} E
$$

coincides with the infimum

$$
\inf _{\mathcal{U}_{\Lambda}} E
$$

and (8) admits a unique minimizer. Here, $\mathcal{U}_{\Lambda}$ is the set of all orientation-preserving maps $\mathbf{u} \in C^{1}(\Omega)^{d}$ that are homeomorphisms from $\bar{\Omega}$ onto $\bar{\Lambda}$.

One can interpret (8) as finding $\overline{\mathbf{u}}$ stable solution of the equations

$$
\left\{\begin{array}{ccc}
-\operatorname{div}\left[\frac{\partial W}{\partial M}(D \overline{\mathbf{u}})\right] & =\mathbf{F} \\
\overline{\mathbf{u}}(\Omega) & = & \Lambda
\end{array}\right.
$$

Uniqueness of a minimizer in (8) clearly implies that, in general, (7) does not admit a minimizer. In fact, sharper conclusions hold for a relaxation of (8): we substitute $\mathcal{U}_{\Lambda}$ by a bigger set $\mathcal{U}_{\Lambda}^{\prime}$ containing maps which may not be smooth. We define $\mathcal{U}_{\Lambda}^{\prime}$ to be the set of all maps $\mathbf{u}$ from $\Omega$ onto $\Lambda$ that are one-to-one almost everywhere and such that $|\operatorname{det} D \mathbf{u}| \neq 0$ almost everywhere in the weak sense. Since it is delicate to define determinants of maps $\mathbf{u} \in \mathcal{U}_{\Lambda}^{\prime}$ we define absolute values of determinants of these maps in the weak sense (see Definition 1.3). We denote by $I$ the extension of $-E$ to $\mathcal{U}_{\Lambda}^{\prime}$. In this new setting, under the assumptions that $\Omega, \Lambda$, are bounded sets and $\mathbf{F} \in L^{1}(\Omega)^{d}$ is one-to-one, $(d-1)$-nondegenerate (see Definition 1.2), we prove that the following problem admits a unique maximizer

$$
\sup _{\mathcal{U}_{\Lambda}^{\prime}} I[\mathbf{u}]
$$

where

$$
I[\mathbf{u}]:=\int_{\Omega}(\mathbf{F} \cdot \mathbf{u}-h(|\operatorname{det} D \mathbf{u}|)) d \mathbf{x} .
$$

If $\overline{\mathbf{u}}$ is the unique maximizer in (10), even if we drop the assumption that $\mathbf{F}$ is $(d-1)$ nondegenerate, then there exists a convex function $\psi_{o}: \mathbf{R}^{d} \rightarrow \mathbf{R}$ such that $\mathbf{F}=$ $D \psi_{o}^{*} \circ \overline{\mathbf{u}}$, and

$$
H(|\operatorname{det} D \overline{\mathbf{u}}|)=\psi_{o}^{*} \circ \overline{\mathbf{u}} .
$$

Here

$$
H(t)=h(t)-t h^{\prime}(t) \quad(t \in \mathbf{R})
$$

and $\psi_{o}^{*}$ is for the Legendre transform of $\psi_{o}$. One can readily check that

$$
H \text { is decreasing and } H(0,+\infty)=\mathbf{R} \text {, }
$$

and so, if $H^{-1}$ is of class $C^{1}$, smoothness of $|\operatorname{det} D \overline{\mathbf{u}}|$ is a straightforward consequence of (11). To understand the relation $\mathbf{F}=D \psi_{o}^{*} \circ \overline{\mathbf{u}}$, one can divide the computation of 
the supremum in (10) into two steps. First, for each function $\alpha>0$, we maximize $\mathbf{u} \rightarrow \int_{\Omega} \mathbf{F} \cdot \mathbf{u} d \mathbf{x}$ over the set of all $\mathbf{u}$ such that $\mathbf{u}(\Omega)=\Lambda$ and $|\operatorname{det} D \mathbf{u}|=\alpha$. Note that this intermediary variational problem is a Monge problem (see [3] and [18] in the case where $\alpha \equiv \chi_{\Omega} d \mathbf{x}$ ), and so the supremum is obtained for a map $\mathbf{u}^{\alpha}$ of the form $D \psi^{\alpha} \circ \mathbf{F}$, where $\psi^{\alpha}$ is a convex function. A sufficient condition for $\psi^{\alpha}$ to be differentiable at $\mathbf{F}(\mathbf{x})$ and thus for $D \psi^{\alpha} \circ \mathbf{F}$ to be well defined at $\mathbf{x}$ is that $\mathbf{F}$ be $(d-1)$-nondegenerate. Formally, if $\alpha_{\infty}$ maximizes the functional $\alpha \rightarrow \int_{\Omega}\left(\mathbf{F} \cdot D \psi^{\alpha} \circ \mathbf{F}-h(\alpha)\right) d \mathbf{x}$ over the set of all $\alpha>0$, then $\overline{\mathbf{u}}=D \psi^{\alpha_{\infty}} \circ \mathbf{F}$ is a maximizer in (10).

Uniqueness of minimizers of $E$ over $\mathcal{U}_{\Lambda}$ and $\mathcal{U}_{o}$ may clearly fail if we don't assume that $\mathbf{F}$ is $(d-1)$-nondegenerate. For instance, let $\mathbf{u}_{o}$ be the identity map, $\mathbf{F} \equiv 0$, and $h(t)=t^{2} / 2+1 /\left(2 t^{2}\right)$. Since $h$ attains it minimum for $t=1$, any map $\mathbf{u} \in \mathcal{U}_{o}$ such that $\operatorname{det} D \mathbf{u}=1$ is a minimizer of $E$ over $\mathcal{U}_{o}$ and $\mathcal{U}_{\Lambda}$ where $\Lambda=\Omega$. Hence, $E$ admits infinitely many minimizers over both sets $\mathcal{U}_{o}$ and $\mathcal{U}_{\Lambda}$. As shown in [17] it is necessary to have that $\operatorname{det} D \mathbf{F}(\mathbf{x}) \geq 0$ for $E$ to admit a minimizer over $\mathcal{U}_{o}$.

Our primary and new contribution is to show that (10) is dual to the minimization problem (13):

$$
\inf _{\mathcal{A}} J[\psi, \phi],
$$

where

$$
J[\psi, \phi]:=\int_{\Omega} \psi(\mathbf{F}(\mathbf{x})) d \mathbf{x}+\int_{\Lambda} \phi(\mathbf{y}) d \mathbf{y},
$$

and $\mathcal{A}$ is the set of all pairs $(\psi, \phi)$ such that $\psi: \mathbf{R}^{d} \rightarrow \mathbf{R} \cup\{+\infty\}$ and $\phi: \operatorname{conv}(\Lambda) \rightarrow$ $\mathbf{R} \cup\{+\infty\}$ are lower semicontinuous, not identically $+\infty$, and

$$
\psi(\mathbf{z})+\alpha \phi(\mathbf{y})+h(\alpha) \geq \mathbf{y} \cdot \mathbf{z}
$$

for all $\mathbf{y} \in \operatorname{conv}(\Lambda)$, all $\mathbf{z} \in \mathbf{R}^{d}$, and all $\alpha>0$. To obtain the above duality result we first show that if $\mu$ is a finite positive measure on $\mathbf{R}^{d}$ of finite moments $M_{o}(\mu)$ and $M_{1}(\mu)$ (see (20)), then

$$
\sup _{\gamma \in \Gamma(\mu)} \bar{I}[\gamma]=\inf _{(\psi, \phi) \in \mathcal{A}} J_{\mu}[\psi, \phi]
$$

where

$$
J_{\mu}[\psi, \phi]:=\int_{\mathbf{R}^{d}} \psi(\mathbf{z}) d \mu(\mathbf{z})+\int_{\Lambda} \phi(\mathbf{y}) d \mathbf{y}
$$

and

$$
\bar{I}[\gamma]:=\int_{C}(\mathbf{y} \cdot \mathbf{z}-h(\alpha)) d \gamma(\alpha, \mathbf{y}, \mathbf{z}) .
$$

Here, $\Gamma[\mu]$ is the set of all Borel measures on $C:=(0,+\infty) \times \mathbf{R}^{d} \times \mathbf{R}^{d}$ such that

$$
\int_{C} f(\mathbf{z}) d \gamma(\alpha, \mathbf{y}, \mathbf{z})=\int_{\mathbf{R}^{d}} f(\mathbf{z}) d \mu(\mathbf{z})
$$

and

$$
\int_{C} \alpha f(\mathbf{y}) d \gamma(\alpha, \mathbf{y}, \mathbf{z})=\int_{\Lambda} f(\mathbf{y}) d \mathbf{y}
$$


for all $f \in C_{o}\left(\mathbf{R}^{d}\right)$.

In fact, one can view $\Gamma(\mu)$ as a set containing $\mathcal{W}$, the set that consists of all Borel maps $\mathbf{w}: \mathbf{R}^{d} \rightarrow \Lambda$ such that the push forward of $\mu$ by $\mathbf{w}$ is absolutely continuous with respect to Lebesgue measure, say, $\mathbf{w} \sharp \mu=d \mathbf{y} / \beta(\mathbf{y})$ for some Borel function $\beta: \Lambda \rightarrow(0,+\infty)$. The inclusion $\mathcal{W} \subset \Gamma(\mu)$ means that we identify $\mathbf{w} \in \mathcal{W}$ to $\gamma^{\mathbf{w}} \in \Gamma(\mu)$, defined by

$$
\int_{C} f(\alpha, \mathbf{y}, \mathbf{z}) d \gamma^{\mathbf{w}}(\alpha, \mathbf{y}, \mathbf{z}):=\int_{\mathbf{R}^{d}} f(\beta(\mathbf{w}(\mathbf{z})), \mathbf{w}(\mathbf{z}), \mathbf{z}) d \mu(\mathbf{z})
$$

for all $f \in C_{o}\left(\mathbf{R} \times \mathbf{R}^{d} \times \mathbf{R}^{d}\right)$. This definition makes sense provided that $\mathbf{w}$ is defined almost everywhere with respect to $\mu$. Observe that if $\mu=\mu_{\mathbf{F}}$ where $\mu_{\mathbf{F}}[A]:=\left|\mathbf{F}^{-1}[A]\right|$ is the $d$-dimensional Lebesgue measure of $\mathbf{F}^{-1}[A]$, then

$$
\bar{I}\left[\gamma^{\mathbf{w}}\right]=I[\mathbf{w} \circ F] .
$$

The plan is to first establish (15) and prove that the variational problems involved admit extremums under the general assumptions that $h$ satisfies (3), (4), and (5) and that $\mu$ is a finite positive measure on $\mathbf{R}^{d}$ whose moments of order one are finite. Next we show that $\bar{I}$ admits a unique maximizer $\gamma_{o}$ over $\Gamma(\mu)$. That maximizer can be parametrized over $\Lambda$ : there is a map $\mathbf{m}: \Lambda \rightarrow \mathbf{R}^{d}$ and a function $\beta: \Lambda \rightarrow \mathbf{R}$, defined $\chi_{\Lambda} d \mathbf{y}$-almost everywhere such that

$$
\int_{C} f(\alpha, \mathbf{y}, \mathbf{z}) d \gamma_{o}(\alpha, \mathbf{y}, \mathbf{z}):=\int_{\Lambda} f(\beta(\mathbf{y}), \mathbf{y}, \mathbf{m}(\mathbf{y})) d \mathbf{y}
$$

for all $f \in C_{o}\left(\mathbf{R} \times \mathbf{R}^{d} \times \mathbf{R}^{d}\right)$. Then, we show that if $\mu_{\mathbf{F}}[A]:=\left|\mathbf{F}^{-1}[A]\right|$ where $\mathbf{F}$ is oneto-one and $(d-1)$-nondegenerate, then every $\gamma_{o}$ maximizing $\bar{I}$ over $\Gamma(\mu)$ is of the form $\gamma^{\mathbf{w}}$ (see (16)). Roughly speaking, $\mu\left[\mathbf{R}^{d} \backslash \mathbf{m}(\Lambda)\right]=0$, $\mathbf{m}$ has an inverse $\mathbf{w}$ defined $\mu$ almost everywhere. We combine (15) and (17) to deduce that $\mathbf{w} \circ F$ maximizes $I$, and that (10) is dual to (13). Simple examples such as $\mathbf{F}(\mathbf{x}) \equiv \mathbf{c}$ and $h(t)=t^{2}+1 / t^{2}$ show that uniqueness of maximizer of $\bar{I}$ over $\Gamma(\mu)$ does not imply uniqueness of maximizer of $I$ over $\mathcal{U}_{\Lambda}^{\prime}$ unless the body force $\mathbf{F}$ is one-to-one and $(d-1)$-nondegenerate.

The remainder of the paper is organized as follows. In section 2 we prove existence of a minimizer $\left(\psi_{o}, \phi_{o}\right)$ of $J_{\mu}$ over $\mathcal{A}$ under the assumptions that $h$ satisfies (3), (4), and (5) and that $\mu$ is a finite positive measure on $\mathbf{R}^{d}$ of finite moments $M_{o}(\mu)$ and $M_{1}(\mu)$. We write the Euler-Lagrange equations corresponding to the variational problem $\inf _{\mathcal{A}} J_{\mu}$ and deduce that if in addition $\mu$ vanishes on $(d-1)$-rectifiable subsets of $\mathbf{R}^{d}$, then there exist a convex function $\psi$ and a positive Borel function $\beta$ such that $D \psi \sharp \mu=d \mathbf{y} / \beta(\mathbf{y})$ and $\gamma_{o}=\gamma^{D \psi}$ maximizes $\bar{I}$ over $\Gamma(\mu)$. It is well known that a convex function is differentiable everywhere except on a $(d-1)$-rectifiable set (see [1]), and so the assumption that $\mu$ vanishes on $(d-1)$-rectifiable subsets of $\mathbf{R}^{d}$ is necessary to guarantee that $D \psi$ exists almost everywhere with respect to $\mu$, so that the measure $\gamma_{o}=\gamma^{D \psi}$ be well-defined. Here, the analytical arguments used to write the EulerLagrange equations corresponding to $\inf _{\mathcal{A}} J_{\mu}$ are similar to the one independently introduced by Caffarelli-Varadhan [5] and the first author [18]. Having $\gamma_{o}$ of the form $\gamma^{D \psi}$ readily yields that the duality (15) holds. By an approximation argument we extend (15) to the case where $\mu$ fails to vanish on $(d-1)$-rectifiable subsets of $\mathbf{R}^{d}$ and still obtain that supports of every maximizers of $\bar{I}$ over $\Gamma(\mu)$ are contained in the graph of a map from $\Lambda$ into $(0,+\infty) \times \mathbf{R}^{d}$. We also show that the maximizer $\gamma_{o}$ of $\bar{I}$ over $\Gamma(\mu)$ is unique. 
In section 3, we assume that the given body force $\mathbf{F}$ belongs to $L^{1}(\Omega)$ and apply results of section 2 with $\mu[A]:=\left|\mathbf{F}^{-1}[A]\right|$ to obtain that (10) is dual to (13). If in addition $\mathbf{F}$ is $(d-1)$-nondegenerate and one-to-one, then $I$ admits a unique maximizer $\overline{\mathbf{u}}$ over $\mathcal{U}_{\Lambda}^{\prime}$. Furthermore, $\overline{\mathbf{u}}$ satisfies $D \psi_{o}^{*} \circ \overline{\mathbf{u}}=\mathbf{F}$ and satisfies the Hamilton-Jacobi equation $H(|\operatorname{det} D \overline{\mathbf{u}}|)=\psi_{o}^{*} \circ \overline{\mathbf{u}}$ for some lower semicontinuous, convex function $\psi_{o}$ : $\mathbf{R}^{d} \rightarrow \mathbf{R}$. Note that if $D \psi_{o}$ is differentiable almost everywhere with respect to $\mu$, then we can conclude that $\overline{\mathbf{u}}=D \psi_{o} \circ \mathbf{F}$. Conversely, we show that if $\overline{\mathbf{u}} \in \mathcal{U}_{\Lambda}^{\prime}, \psi_{o}: \mathbf{R}^{d} \rightarrow \mathbf{R}$ is a lower semicontinuous, convex function such that $H(|\operatorname{det} D \overline{\mathbf{u}}|)=\psi_{o}^{*} \circ \overline{\mathbf{u}}$ and $\mathbf{F}=$ $D \psi_{o}^{*} \circ \overline{\mathbf{u}}$, then $\overline{\mathbf{u}}$ is the unique maximizer of $I$ over $\mathcal{U}_{\Lambda}^{\prime}$.

In section 4, using Caffarelli's regularity results on smoothness of convex potentials [4], [5], [6], we prove that if $\mathbf{F}$ and $\operatorname{det} D \mathbf{F}$ are of class $C^{1}$, if $\Lambda$ and $\mathbf{F}(\Omega)$ are convex sets, then $\overline{\mathbf{u}}$ is of class $C^{1}$ and is the unique minimizer of $E$ over $\mathcal{U}_{\Lambda}$. A corollary of this result is that given a diffeomorphism $\mathbf{u}_{o}$ of $\bar{\Omega}$ onto $\bar{\Lambda}$, the infima inf $\mathcal{U}_{\Lambda} E$ and $\inf _{\mathcal{U}_{o}} E$ coincide.

Four appendices are also provided. In Appendix A, we review basic facts about convex functions and study needed properties of the transformations introduced in Definition 1.6, $\phi \rightarrow \phi^{\sharp}, \psi \rightarrow \psi_{\sharp}$ from the set of real-valued functions to the set of convex functions. In Appendix $\mathrm{C}$, we state that every one-to-one map $\mathbf{u} \in \mathcal{U}_{\Lambda}$ of class $C^{1}(\Omega) \cap C(\bar{\Omega})$ such that $\operatorname{det} D \mathbf{u}+\frac{1}{\operatorname{det} D \mathbf{u}}$ is bounded is a pointwise limit of a sequence of one-to-one maps $\left(\mathbf{u}_{n}\right) \subset \mathcal{U}_{o}$ of class $C^{1}(\Omega) \cap C(\bar{\Omega})$ with $\operatorname{det} D \mathbf{u}_{n}=\operatorname{det} D \mathbf{u}$. This approximation result is used in section 4 to prove that the infima inf $\mathcal{U}_{\Lambda} E$ and $\inf _{\mathcal{U}_{o}} E$ coincide. In Appendix D we recall facts on existence and smoothness of optimal maps in the Monge problem.

We next summarize the main results of the paper.

TheOREM 0.1 (main results). Suppose that $\Omega, \Lambda \subset \mathbf{R}^{d}$ are bounded open sets, that (3), (4), and (5) hold, and that $\mathbf{F} \in L^{1}(\Omega)^{d}$ is a Borel map. Then we have the following.

(i) Duality. $J$ admits a minimizer $\left(\psi_{o}, \phi_{o}\right)$ over $\mathcal{A}$ and we have that $\inf _{\mathcal{A}} J[\psi, \phi]=$ $\sup _{\mathcal{U}_{\wedge}^{\prime}} I[\mathbf{u}]$.

(ii) Uniqueness of a minimizer. If in addition $\mathbf{F}$ is one-to-one almost everywhere with respect to the $d$-dimensional Lebesgue measure and $\left|\mathbf{F}^{-1}(N)\right|=0$ whenever $N$ is $(d-1)$-rectifiable, then $I$ admits a unique maximizer $\overline{\mathbf{u}}$ over $\mathcal{U}_{\Lambda}^{\prime}$; we also have that $\overline{\mathbf{u}}=D \psi_{o} \circ \mathbf{F}$, and $H(|\operatorname{det} D \overline{\mathbf{u}}|)=\psi_{o}^{*} \circ \overline{\mathbf{u}}$, where $\left(\psi_{o}, \phi_{o}\right)$ minimizes $J$ over $\mathcal{A}$.

(iii) Smoothness of the minimizer. Assume in addition that $\Omega$ is connected, its boundary $\partial \Omega$ is Lipschitz, and $\Lambda, \mathbf{F}(\Omega)$ are convex. If $\mathbf{F}$ and $\operatorname{det} D \mathbf{F}$ belong to $C^{1}(\bar{\Omega})^{d}$ and $\operatorname{det} D \mathbf{F}>0$ on $\bar{\Omega}$, then $\overline{\mathbf{u}} \in \mathcal{U}_{\Lambda} \cap C^{0, s}(\bar{\Omega})^{d}, 0<\operatorname{det} D \overline{\mathbf{u}} \in C^{0, s}(\bar{\Omega}) \cap C^{1}(\Omega)$ for all $0<s<1, \overline{\mathbf{u}}$ is the unique minimizer of $E$ over $\mathcal{U}_{\Lambda}$. Furthermore, we have that $-\operatorname{div}\left[\frac{\partial W}{\partial M}(D \overline{\mathbf{u}})\right]=\mathbf{F}$ in $\Omega$ in the weak sense.

Proof. Parts (i) and (ii) follow from Theorem 3.1, and (iii) is a consequence of Theorem 4.1.

Simple calculations show that the duality result obtained in Theorem 0.1 is

$$
\inf _{\mathcal{U}_{\Lambda}^{\prime}}\left\{\int_{\Omega}(h(\operatorname{det} D \mathbf{u})-\mathbf{F} \cdot \mathbf{u}) d \mathbf{x}\right\}=\sup _{b}\left\{\int_{\Omega} L_{b}(\mathbf{F}(\mathbf{x})) d \mathbf{x}+\int_{\Lambda} b(\mathbf{y}) d \mathbf{y}\right\},
$$

where the supremum is performed over the set of all upper semicontinuous functions $b: \mathbf{R}^{d} \rightarrow \mathbf{R}$ and

$$
L_{b}(\mathbf{z}):=\inf _{\mathbf{y} \in \operatorname{conv}(\Lambda)}\left\{-\mathbf{y} \cdot \mathbf{z}-h^{*}(b(\mathbf{y}))\right\}
$$


1. Notations and definitions. For the convenience of the reader we collect together some of the notation introduced throughout the text.

- If $\Omega \subset \mathbf{R}^{d}$, then $\bar{\Omega}$ denotes the closure of $\Omega$.

- $B_{R}$ is the closed ball of center 0 and radius $R>0$.

- $|A|$ stands for the $d$-dimensional Lebesgue measure of the set $A \subset \mathbf{R}^{d}$, and $\int_{\mathbf{R}^{d}} G d \mathbf{x}$ is the Lebesgue integral of $G$.

- If $\mu$ is a Borel measure on $\mathbf{R}^{d}$, then we denote by $\operatorname{spt} \mu$ the support of $\mu$, which refers to the smallest closed set $K$ such that $\mu\left[\mathbf{R}^{d} \backslash K\right]=0$. If $\mu$ is absolutely continuous with respect to the $d$-dimensional Lebesgue measure and $\mu[A]=\int_{A} f d \mathbf{x}$ for $A \subset \mathbf{R}^{d}$ Borel, then we write $\mu=f d \mathbf{x}$.

- If $\mu$ is a Borel measure on $\mathbf{R}^{d}$ and $\mathbf{v}: \mathbf{R}^{d} \rightarrow \mathbf{R}^{m}$ is a Borel map, then we define $\mathbf{v}_{\#} \mu$ to be the Borel measure on $\mathbf{R}^{m}$ given by $\mathbf{v}_{\#} \mu[B]:=\mu\left[\mathbf{v}^{-1}(B)\right]$ for $B \subset \mathbf{R}^{m}$.

- The characteristic function of $A \subset \mathbf{R}^{d}$ is denoted by $\chi_{A}$.

- If $\psi: \mathbf{R}^{d} \rightarrow \mathbf{R} \cup\{+\infty\}$ is not identically $+\infty$, then the Legendre-Fenchel transform of $\psi$ is the convex, lower semicontinuous function $\psi^{*}: \mathbf{R}^{d} \rightarrow \mathbf{R} \cup\{+\infty\}$ defined by

$$
\psi^{*}(\mathbf{y}):=\sup _{\mathbf{x} \in \mathbf{R}^{d}}\{\mathbf{x} \cdot \mathbf{y}-\psi(\mathbf{x})\} .
$$

- The subdifferential of a convex function $\psi: \mathbf{R}^{d} \rightarrow \mathbf{R} \cup\{+\infty\}$ is the set $\partial \psi \subset$ $\mathbf{R}^{d} \times \mathbf{R}^{d}$ consisting of all $(\mathbf{x}, \mathbf{y})$ satisfying

$$
\psi(\mathbf{z})-\psi(\mathbf{x}) \geq \mathbf{y} \cdot(\mathbf{z}-\mathbf{x}) \quad \text { for all } \mathbf{z} \in \mathbf{R}^{d} .
$$

If $(\mathbf{x}, \mathbf{y}) \in \partial \psi$, we may also write $\mathbf{y} \in \partial \psi(\mathbf{x})$. Recall $\mathbf{x} \in \partial \psi^{*}(\mathbf{y})$ whenever $\mathbf{y} \in \partial \psi(\mathbf{x})$, while the converse also holds true if $\psi$ is convex lower semicontinuous. In that case $\partial \psi$ is a closed set. In general, the set $\partial \psi(\mathbf{x}) \subset \mathbf{R}^{d}$ is closed and convex.

- id stands for the identity map $\operatorname{id}(\mathbf{x})=\mathbf{x}$.

- We denote the set of all $d \times d$ matrices whose entries are real numbers by $\mathbf{R}^{d \times d}$.

- We denote the set of all homeomorphism from $A \subset \mathbf{R}^{d}$ onto $B \subset \mathbf{R}^{d}$ by $\operatorname{Diff}^{0}(A, B)$. If $k \geq 1$ is an integer, $\Omega, \Lambda \subset \mathbf{R}^{d}$ are open, then $\operatorname{Diff}^{k}(\Omega, \Lambda)$ is the set of all maps $\mathbf{v} \in \operatorname{Diff} f^{0}(\Omega, \Lambda)$ such that $\mathbf{v} \in C^{k}(\Omega)^{d}$ and $\mathbf{v}^{-1} \in C^{k}(\Lambda)^{d}$. We denote the set of all maps $\mathbf{v} \in \operatorname{Diff}^{0}(\bar{\Omega}, \bar{\Lambda})$ such that $\mathbf{v}$ is of class $C^{k}$ in a neighborhood of $\bar{\Omega}$ and $\mathbf{v}^{-1}$ is of class $C^{k}$ in a neighborhood of $\bar{\Lambda}$ by $\operatorname{Diff} f^{k}(\bar{\Omega}, \bar{\Lambda})$.

- We define $\mathcal{U}_{o}$ to be the set of all continuous maps $\mathbf{u}$ from $\bar{\Omega}$ onto $\bar{\Lambda}$ that are in $C^{1}(\Omega)^{d}$, such that $\operatorname{det} D \mathbf{u}>0, \mathbf{u}$, and $\mathbf{u}_{o}$ coincide on $\partial \Omega . \mathcal{U}_{\Lambda}$ is the set of all orientation-preserving maps $\mathbf{u} \in C^{1}(\Omega)^{d}$ that are homeomorphisms from $\bar{\Omega}$ onto $\bar{\Lambda}$. $\mathcal{U}_{\Lambda}^{\prime}$ is the set of all maps $\mathbf{u}$ from $\Omega$ onto $\Lambda$ that are one-to-one almost everywhere and such that $|\operatorname{det} D \mathbf{u}| \neq 0$ almost everywhere in the weak sense.

- We define $\mathcal{A}$ to be the set of all pairs of functions $(\psi, \phi)$ such that $\psi: \mathbf{R}^{d} \rightarrow$ $\mathbf{R} \cup\{+\infty\}, \phi: \operatorname{conv}(\Lambda) \rightarrow \mathbf{R} \cup\{+\infty\}$ are lower semicontinuous, not identically $+\infty$, and $\psi(\mathbf{z})+\alpha \phi(\mathbf{y})+h(\alpha) \geq \mathbf{y} \cdot \mathbf{z}$ for all $\mathbf{y} \in \operatorname{conv}(\Lambda), \mathbf{z} \in \mathbf{R}^{d}$, and all $\alpha>0$.

We recall definitions needed in that which follows.

Definition 1.1. Let $A, B \subset \mathbf{R}^{d}$. We say that $\mathbf{v}: A \rightarrow B$ is one-to-one almost everywhere from $A$ onto $B$ (with respect to the d-dimensional Lebesgue measure) if $|B \backslash \mathbf{v}(A)|=0$, if there exists a set $N \subset A$ such that $|N|=0$, and if the restriction of $\mathbf{v}$ to $A \backslash N$ is one-to-one. By abuse of language we omit the expression "with respect to the d-dimensional Lebesgue measure."

Definition 1.2. Let $A, B \subset \mathbf{R}^{d}$. We say that a Borel map $\mathbf{v}: A \rightarrow B$ is nondegenerate if $\left|\mathbf{v}^{-1}(N)\right|=0$ whenever $|N|=0$. We say that $\mathbf{v}$ is $(d-1)$-nondegenerate if $\left|\mathbf{v}^{-1}(N)\right|=0$ whenever $N$ is $(d-1)$-rectifiable. 
Recall that $N \subset \mathbf{R}^{d}$ is $(d-1)$-rectifiable if $N$ is a countable union of $(d-1)$ hypersurfaces of class $C^{1}$, union a set of zero $(d-1)$-dimensional Hausdorff measure.

Definition 1.3. Let $A, B \subset \mathbf{R}^{d}$, and let $\beta_{o} \in L^{1}(A), \beta_{1} \in L^{1}(B)$ be nonnegative functions. Let $\mathbf{v}: A \rightarrow B$ be a one-to-one almost everywhere Borel map from $A$ onto $B$. We say that $\beta_{1}(\mathbf{v}(\mathbf{x}))|\operatorname{det} D \mathbf{v}(\mathbf{x})|=\beta_{o}(\mathbf{x})$ in $A$ in the weak sense if

$$
\int_{A} \varphi(\mathbf{v}(\mathbf{x})) \beta_{o}(\mathbf{x}) d \mathbf{x}=\int_{B} \varphi(\mathbf{y}) \beta_{1}(\mathbf{y}) d \mathbf{y}
$$

for all $\varphi \in C_{o}\left(\mathbf{R}^{d}\right)$.

Remark 1.4. Note that if $\mathbf{v}$ is one-to-one almost everywhere, and if $\left|\mathbf{v}^{-1}[C]\right|=|C|$ for every Borel set $C$, then $|\operatorname{det} D \mathbf{v}|=1$ in the weak sense although $D \mathbf{v}$ may not exist.

Definition 1.5. Let $\mu$ and $\nu$ be two Borel measures on $\mathbf{R}^{d}$. We say that the Borel map $\mathbf{v}: \mathbf{R}^{d} \rightarrow \mathbf{R}^{d}$ pushes $\mu$ forward to $\nu$ and we write $\mathbf{v} \sharp \mu=\nu$ if $\mu\left[\mathbf{v}^{-1}(B)\right]=\nu[B]$ for all Borel sets $B \subset \mathbf{R}^{d}$.

DeFinition 1.6. If $\phi$ and $\psi$ are two real valued functions of subsets of $\mathbf{R}^{d}$ into $\mathbf{R} \cup\{+\infty\}$, then we define $\phi^{\sharp}$ and $\psi_{\sharp}$ to be the following convex functions of $\mathbf{R}^{d}$ into $\mathbf{R} \cup\{+\infty\}:$

$$
\phi^{\sharp}(\mathbf{z}):=\sup _{\mathbf{y} \in \operatorname{conv}(\Lambda)}\left\{\mathbf{y} \cdot \mathbf{z}+h^{*}(-\phi(\mathbf{y}))\right\} \quad \text { and } \quad \psi_{\sharp}(\mathbf{y}):=\sup _{\alpha>0}\left\{\frac{\psi^{*}(\mathbf{y})-h(\alpha)}{\alpha}\right\} .
$$

2. An auxiliary variational problem: Duality. Throughout this section we assume that $\Lambda \subset \mathbf{R}^{d}$ is an open bounded set whose closure is contained in the closed ball $B_{R_{o}}$ of center 0 and radius $R_{o}$. We assume that $h$ satisfies (3), (4), (5) and $\mu$ is a finite positive measure on $\mathbf{R}^{d}$ of finite moments $M_{o}(\mu)$ and $M_{1}(\mu)$, where

$$
M_{o}(\mu):=\mu\left[\mathbf{R}^{d}\right]<+\infty, \quad M_{1}(\mu):=\int_{\mathbf{R}^{d}}|\mathbf{z}| d \mu(\mathbf{z})<+\infty .
$$

We define

$$
J_{\mu}[\psi, \phi]:=\int_{\mathbf{R}^{d}} \psi(\mathbf{z}) d \mu(\mathbf{z})+\int_{\Lambda} \phi(\mathbf{y}) d \mathbf{y}
$$

and

$$
\bar{I}[\gamma]:=\int_{C}(\mathbf{y} \cdot \mathbf{z}-h(\alpha)) d \gamma(\alpha, \mathbf{y}, \mathbf{z}),
$$

where $C$ is the set $(0, \infty) \times \mathbf{R}^{d} \times \mathbf{R}^{d}$. Let $\Gamma[\mu]$ be the set of all Borel measures on $C$ such that

$$
\int_{C} f(\mathbf{z}) d \gamma(\alpha, \mathbf{y}, \mathbf{z})=\int_{\mathbf{R}^{d}} f(\mathbf{z}) d \mu(\mathbf{z})
$$

and

$$
\int_{C} \alpha f(\mathbf{y}) d \gamma(\alpha, \mathbf{y}, \mathbf{z})=\int_{\Lambda} f(\mathbf{y}) d \mathbf{y}
$$

for all $f \in C_{o}\left(\mathbf{R}^{d}\right)$. Observe that for every $(\psi, \phi) \in \mathcal{A}$ and every $\gamma \in \Gamma(\mu)$ we have that

$$
J_{\mu}[\psi, \phi]=\int_{C}(\psi(\mathbf{z})+\alpha \phi(\mathbf{y})) d \gamma \geq \int_{C}(\mathbf{y} \cdot \mathbf{z}-h(\alpha)) d \gamma=\bar{I}[\gamma]
$$


and so

$$
\sup _{\Gamma(\mu)} \bar{I}[\gamma] \leq \inf _{\mathcal{A}} J_{\mu}[\psi, \phi] .
$$

We establish the reverse inequality in this section.

Remark 2.1. Note that if $(\psi, \phi) \in \mathcal{A}$, then we have that

$$
\psi^{-}(\mathbf{z}) \leq R_{o}|\mathbf{z}|+|h(1)|+\inf _{\operatorname{conv}(\Lambda)} \phi^{+}
$$

for all $\mathbf{z} \in \mathbf{R}^{d}$ and

$$
\phi^{-}(\mathbf{y}) \leq|\mathbf{y}||\mathbf{z}|+|h(1)|+\psi^{+}(\mathbf{z})
$$

for all $\mathbf{y} \in \operatorname{conv}(\Lambda), \mathbf{z} \in \mathbf{R}^{d}$. Combining (20), (22), and (23) we deduce that both $\int_{\mathbf{R}^{d}} \psi(\mathbf{z}) d \mu(\mathbf{z}), \int_{\Lambda} \phi(\mathbf{y}) d \mathbf{y}$ exist although they may be $+\infty$ and $J_{\mu}[\psi, \phi]$ is well-defined.

Lemma 2.2. The set $\mathcal{A}$ contains at least an element $(\psi, \phi)$. Also, there exists a constant $c_{a}$ depending only on $h, \Lambda, M_{o}[\mu]$ such that

(i) $\left|\inf _{\mathcal{A}} J_{\mu}\right| \leq c_{a}\left(1+M_{1}[\mu]\right)$;

(ii) if $\psi, \phi$ are convex and $\left|J_{\mu}[\psi, \phi]-\inf _{\mathcal{A}} J_{\mu}\right| \leq 1$, then

$$
\int_{\Lambda}|\phi(\mathbf{y})| d \mathbf{y} \text { and } \int_{\mathbf{R}^{d}}|\psi(\mathbf{z})| d \mu(\mathbf{z}) \leq c_{a}\left(1+M_{1}[\mu]\right)
$$

(iii) if in addition $\operatorname{Lip}(\psi) \leq R_{o}$, then we have that

$$
\psi(\mathbf{z}) \leq R_{o}|\mathbf{z}|+R R_{o}+\frac{c_{a}}{\mu\left[B_{R}\right]}\left(1+M_{1}[\mu]\right) \quad\left(\mathbf{z} \in \mathbf{R}^{d}\right) .
$$

Proof. Step 1 . The set $\mathcal{A}$ is nonempty since it contains $\left(\psi_{o}, \phi_{o}\right)$, where $\phi_{o}(\mathbf{y}):=1$ on $\operatorname{conv}(\Lambda), \psi_{o}(\mathbf{z}):=R_{o}|\mathbf{z}|-c$ on $\mathbf{R}^{d}$, and $c:=\inf _{\alpha>0}\{h(\alpha)+\alpha\}$. We deduce that

$$
\inf _{\mathcal{A}} J_{\mu} \leq J_{\mu}\left[\psi_{o}, \phi_{o}\right] \leq|\Lambda|+R_{o} M_{1}[\mu]-c M_{o}[\mu] .
$$

If $(\psi, \phi) \in \mathcal{A}$, then

$$
J_{\mu}[\psi, \phi] \geq-\left(\alpha \phi\left(\mathbf{y}_{o}\right)+h(\alpha)\right) M_{o}[\mu]-R_{o} M_{1}[\mu]+\int_{\Lambda} \phi(\mathbf{y}) d \mathbf{y}
$$

for all $\alpha>0$ and all $\mathbf{y}_{o} \in \Lambda$. Setting $\alpha:=|\Lambda| / M_{o}[\mu]$ in (25) and using (24) we have that

$$
\left|\inf _{\mathcal{A}} J_{\mu}\right| \leq c_{1}
$$

where $c_{1}:=|\Lambda|+R_{o} M_{1}[\mu]+h\left(|\Lambda| / M_{o}[\mu]\right) M_{o}[\mu]$.

Step 2. Let $(\psi, \phi) \in \mathcal{A}$ be such that $\left|J_{\mu}[\psi, \phi]-\inf _{\mathcal{A}} J_{\mu}\right| \leq 1$. In light of (25) we have that

$$
\int_{\Lambda} \phi(\mathbf{y}) d \mathbf{y} \leq 1+\inf _{\mathcal{A}} J_{\mu}+\left(\alpha \phi\left(\mathbf{y}_{o}\right)+h(\alpha)\right) M_{o}[\mu]+R_{o} M_{1}[\mu]
$$

for all $\alpha>0$ and all $\mathbf{y}_{o} \in \Lambda$. Choosing $\alpha$ and $\mathbf{y}_{o}$ appropriately in (27) we have that

$$
\left|\int_{\Lambda} \phi(\mathbf{y}) d \mathbf{y}\right| \leq c_{2}\left(1+M_{1}[\mu]\right)
$$


where $c_{2}$ is a constant depending only on $h, \Lambda, M_{o}[\mu]$. Combining (26) and (28) we deduce that there exists a constant $c_{3}$ depending only on $h, \Lambda$, and $M_{o}[\mu]$ such that

$$
\left|\int_{\Lambda} \phi(\mathbf{y}) d \mathbf{y}\right|,\left|\int_{\mathbf{R}^{d}} \psi(\mathbf{z}) d \mu(\mathbf{z})\right| \leq c_{3}\left(1+M_{1}[\mu]\right) .
$$

Step 3. Assume that $(\psi, \phi) \in \mathcal{A}, \phi$ is convex on $\operatorname{conv}(\Lambda), \psi$ is convex on $\mathbf{R}^{d}$, and $\left|J_{\mu}[\psi, \phi]-\inf _{\mathcal{A}} J_{\mu}\right| \leq 1$. In light of (29) there exists $\mathbf{z}_{o} \in \mathbf{R}^{d}$ such that

$$
\left|\psi\left(\mathbf{z}_{o}\right)\right| \leq c_{3}\left(1+M_{1}[\mu]\right) / \mu\left[\mathbf{R}^{d}\right] .
$$

Integrating (23) over $\mathbf{R}^{d}$ we have that

$$
M_{o}[\mu] \phi^{-}(\mathbf{y}) \leq|\mathbf{y}| M_{1}[\mu]+|h(1)| M_{o}[\mu]+\int_{\mathbf{R}^{d}} \psi^{+}(\mathbf{z}) d \mu(\mathbf{z})
$$

for all $\mathbf{y} \in \operatorname{conv}(\Lambda)$. Either $\inf _{\operatorname{conv}(\Lambda)} \phi^{+}>0$, in which case

$$
\phi^{-} \equiv 0 \quad \text { on } \operatorname{conv}(\Lambda),
$$

or $\inf _{\operatorname{conv}(\Lambda)} \phi^{+}=0$, in which case (22) and (29) imply that there exists a constant $c_{4}$ depending only on $h, \Lambda$, and $M_{o}[\mu]$ such that

$$
\int_{\mathbf{R}^{d}}|\psi(\mathbf{z})| d \mu(\mathbf{z}) \leq c_{4}\left(1+M_{1}[\mu]\right)
$$

which, combined with (31), yields

$$
M_{o}[\mu] \phi^{-}(\mathbf{y}) \leq|\mathbf{y}| M_{1}[\mu]+|h(1)| M_{o}[\mu]+c_{4}\left(1+M_{1}[\mu]\right)
$$

for all $\mathbf{y} \in \operatorname{conv}(\Lambda)$. Using (32) and (33) we deduce that in any case, there exists a constant $c_{5}$ depending only on $h, \Lambda$, and $M_{o}[\mu]$ such that

$$
\phi^{-}(\mathbf{y}) \leq c_{5}\left(1+M_{1}[\mu]\right)
$$

for all $\mathbf{y} \in \operatorname{conv}(\Lambda)$. In light of $(29)$ and (34) we have that there exists a constant $c_{6}$ depending only on $h, \Lambda$, and $M_{o}[\mu]$ such that

$$
\int_{\Lambda}|\phi(\mathbf{y})| d \mathbf{y} \leq c_{6}\left(1+M_{1}[\mu]\right) .
$$

Since $\phi$ is convex, (35) implies that for each $K \subset \Lambda$ compact set, there exists a constant $c_{K}$ depending only on $h, \Lambda, M_{o}[\mu]$, and $K$ such that (see [13, p. 236])

$$
|\phi|_{L^{\infty}(K)}+|D \phi|_{L^{\infty}(K)} \leq c_{K}\left(1+M_{1}[\mu]\right) .
$$

Now, (22) and (36) imply that there exists a constant $c_{7}$ depending only on $h, \Lambda$, and $M_{o}[\mu]$ such that

$$
\psi^{-}(\mathbf{z}) \leq R_{o}|\mathbf{z}|+c_{7}\left(1+M_{1}[\mu]\right)
$$

for all $\mathbf{z} \in \mathbf{R}^{d}$. By (29) and (37) we have that there exists a constant $c_{8}$ depending only on $h, \Lambda$, and $M_{o}[\mu]$ such that

$$
\int_{\mathbf{R}^{d}}|\psi(\mathbf{z})| d \mu(\mathbf{z}) \leq c_{8}\left(1+M_{1}[\mu]\right)
$$


This concludes the proof of (ii).

Step 4. By (38),

$$
\mu\left[B_{R}\right] \inf _{B_{R}}|\psi| \leq c_{8}\left(1+M_{1}[\mu]\right),
$$

and so, if in addition $\operatorname{Lip}(\psi) \leq R_{o}$, we readily obtain (iii). This concludes the proof of the lemma.

Proposition 2.3. Suppose that $\mu$ satisfies (20) such that $\left(\mu_{n}\right)$ is a sequence of Borel measures, that $M_{o}\left[\mu_{n}\right]=M_{o}[\mu](n=1,2, \ldots)$, that $\left(\mu_{n}\right)$ converges weak $*$ to $\mu$, and that $\left(M_{1}\left[\mu_{n}\right]\right)$ converges to $M_{1}[\mu]$. Then the following hold:

(i) There exists $\left(\psi_{\mu}, \phi_{\mu}\right) \in \mathcal{A}$ minimizing $J_{\mu}$ over $\mathcal{A}$, and

$$
\inf _{\mathcal{A}} J_{\mu} \leq \liminf _{n \rightarrow+\infty}\left(\inf _{\mathcal{A}} J_{\mu_{n}}\right) .
$$

(ii) We have that $\lim \sup _{n \rightarrow+\infty}\left(\sup _{\Gamma\left(\mu_{n}\right)} \bar{I}\right) \leq \sup _{\Gamma(\mu)} \bar{I}$.

(iii) If $\sup _{\Gamma(\mu)} \bar{I} \neq-\infty$, then there exists $\gamma_{\mu} \in \Gamma(\mu)$ maximizing $\bar{I}$ over $\Gamma(\mu)$.

Proof. Step 1. We shall show in Step 5 that (i) is a direct consequence of the following statement: If $\left(f_{n}, g_{n}\right) \in \mathcal{A}$ is such that $\left|\inf _{\mathcal{A}} J_{\mu_{n}}-J_{\mu_{n}}\left(f_{n}, g_{n}\right)\right| \leq 1 / n$, then there exists $\left(\psi_{\mu}, \phi_{\mu}\right) \in \mathcal{A}$ such that

$$
J_{\mu}\left(\psi_{\mu}, \phi_{\mu}\right) \leq \liminf _{n \rightarrow+\infty} J_{\mu_{n}}\left(f_{n}, g_{n}\right) \quad(n=1,2, \ldots) .
$$

To proceed, let $R_{1}>0$ be such that

$$
\mu\left[\operatorname{int}\left(B_{R_{1}}\right)\right]>1 / 2 \mu\left[\mathbf{R}^{d}\right] .
$$

Note that since $\left(\mu_{n}\right)$ converges weak $*$ to $\mu$, in light of (40) we may assume without loss of generality that (see [13, p. 59])

$$
\mu_{n}\left[\operatorname{int}\left(B_{R_{1}}\right)\right]>1 / 2 M_{o}[\mu]=1 / 2 M_{o}\left[\mu_{n}\right]
$$

for all $n=1,2, \ldots$ Define

$$
\phi_{n}:=\left(f_{n}\right)_{\sharp}, \quad \psi_{n}:=\left(\phi_{n}\right)^{\sharp} .
$$

By Lemma A.1 (ii)-(iii) $\psi_{n}$ and $\phi_{n}$ are convex functions, $\psi_{n} \leq f_{n}, \phi_{n} \leq g_{n}$, and

$$
\operatorname{Lip}\left(\psi_{n}\right) \leq R_{o} ;
$$

hence

$$
J_{\mu_{n}}\left(\psi_{n}, \phi_{n}\right) \leq J_{\mu_{n}}\left(f_{n}, g_{n}\right)
$$

for all $n=1,2, \ldots$. Since in addition $\left|\inf _{\mathcal{A}} J_{\mu_{n}}-J_{\mu_{n}}\left(\psi_{n}, \phi_{n}\right)\right| \leq 1 / n$, by Lemma 2.2 and (41) there exists a constant $\bar{c}>0$ independent of $n$ such that

$$
\int_{\Lambda}\left|\phi_{n}(\mathbf{y})\right| d \mathbf{y} \leq \bar{c}
$$

and

$$
\left|\psi_{n}(\mathbf{z})\right| \leq R_{o}|\mathbf{z}|+\bar{c} \quad\left(\mathbf{z} \in \mathbf{R}^{d}\right) .
$$


Using (45) we deduce that the sequence $\left(\psi_{n}\right)$ is bounded in $W^{1, \infty}\left(B_{R^{\prime}}\right)$ for every $R^{\prime}>0$. Since $\psi_{n}$ is convex, we may find a subsequence of $\left(\psi_{n}\right)$ that we still label $\left(\psi_{n}\right)$, converging in $L_{l o c}^{\infty}\left(\mathbf{R}^{d}\right)$ to a convex function $\psi_{\mu}: \mathbf{R}^{d} \rightarrow \mathbf{R}$. One can readily check the following claims.

Step 2. Claim. We have that

$$
\limsup _{n \rightarrow+\infty} \int_{B_{R}^{c}}\left(R_{o}|\mathbf{z}|+\bar{c}\right) d \mu_{n}(\mathbf{z}) \leq \int_{B_{R-2}^{c}}\left(R_{o}|\mathbf{z}|+\bar{c}\right) d \mu(\mathbf{z})
$$

for all $R>2$.

Step 3. Claim. We have that $\lim _{n \rightarrow+\infty} \int_{\mathbf{R}^{d}}\left|\psi_{n}-\psi_{\mu}\right| d \mu_{n}=0$.

We next prove the following.

Step 4. Claim. We have that $\liminf _{n \rightarrow+\infty} \int_{\mathbf{R}^{d}} \psi_{\mu} d \mu_{n} \geq \int_{\mathbf{R}^{d}} \psi_{\mu} d \mu$.

Proof: For $R>1$ let $l_{R}: \mathbf{R} \rightarrow[0,1]$ be of class $C^{\infty}$ such that

$$
l_{R}(t)=\left\{\begin{array}{llc}
1 & \text { if } & |t| \leq R-1 \\
0 & \text { if } & |t| \geq R
\end{array}\right.
$$

We have that

$$
\chi_{B_{R}^{c}} \leq 1-l_{R}(|\mathbf{z}|) \leq \chi_{B_{R-2}^{c}} .
$$

Because $\left(\mu_{n}\right)$ converges weak $*$ to $\mu$ and $\left(M_{1}\left[\mu_{n}\right]\right)$ converges to $M_{1}[\mu]$, using (45) and (47) we have that

$$
\begin{aligned}
\liminf _{n \rightarrow+\infty} \int_{\mathbf{R}^{d}} \psi_{\mu} d \mu_{n} & \geq \int_{\mathbf{R}^{d}} \psi_{\mu} l_{R} d \mu-\int_{\mathbf{R}^{d}}\left(R_{o}|\mathbf{z}|+\bar{c}\right)\left(1-l_{R}(|\mathbf{z}|)\right) d \mu \\
& \geq \int_{\mathbf{R}^{d}} \psi_{\mu} l_{R} d \mu-\int_{B_{R-2}^{c}}\left(R_{o}|\mathbf{z}|+\bar{c}\right) d \mu
\end{aligned}
$$

Letting $R$ go to $+\infty$ in (48) we conclude the proof of Claim 4 .

Now, combining Claims 3 and 4 we have that

$$
\int_{\mathbf{R}^{d}} \psi_{\mu} d \mu \leq \liminf _{n \rightarrow+\infty} \int_{\mathbf{R}^{d}} \psi_{n} d \mu_{n}
$$

Similarly, since $\phi_{n}$ is convex (44) implies that there exists a convex function $\phi_{\mu}$ : $\operatorname{conv}(\Lambda) \rightarrow \mathbf{R} \cup\{+\infty\}$ such that up to a subsequence, $\left(\phi_{n}\right)$ converges pointwise to $\phi_{\mu}$ in $\Lambda$ and

$$
\int_{\Lambda} \phi_{\mu} d \mathbf{y} \leq \liminf _{n \rightarrow+\infty} \int_{\Lambda} \phi_{n} d \mathbf{y}
$$

Because $\left(\psi_{n}, \phi_{n}\right) \in \mathcal{A}$, we obtain that $\left(\psi_{\mu}, \phi_{\mu}\right) \in \mathcal{A}$. Thanks to (43), (49), and (50) we have that

$$
\inf _{\mathcal{A}} J_{\mu} \leq J_{\mu}\left(\psi_{\mu}, \phi_{\mu}\right) \leq \liminf _{n \rightarrow+\infty} J_{\mu_{n}}\left(f_{n}, g_{n}\right)
$$

which proves (39).

Step 5. Taking $\mu_{n} \equiv \mu$ for all $n$ in (51) we have that there exists $\left(\psi_{\mu}, \phi_{\mu}\right) \in \mathcal{A}$ minimizing $J_{\mu}$ over $\mathcal{A}$. Next, assuming $\left(f_{n}, g_{n}\right)$ minimizes $J_{\mu_{n}}$ over $\mathcal{A},(51)$ implies that $\inf _{\mathcal{A}} J_{\mu} \leq \liminf _{n \rightarrow+\infty}\left(\inf _{\mathcal{A}} J_{\mu_{n}}\right)$ which completes the proof of (i). 
If $\lim \sup _{n \rightarrow+\infty}\left(\sup _{\Gamma\left(\mu_{n}\right)}\right) \bar{I}=-\infty$, then (ii) is straightforward to obtain.

Step 6. Now we prove (ii). If lim $\sup _{n \rightarrow+\infty}\left(\sup _{\Gamma\left(\mu_{n}\right)}\right) \bar{I}=-\infty$, then (ii) is straightforward to obtain. Therefore we may assume without loss of generality that

$$
\limsup _{n \rightarrow+\infty}\left(\sup _{\Gamma\left(\mu_{n}\right)} \bar{I}\right)>-\infty .
$$

Note first that since by $(21) \sup _{\Gamma\left(\mu_{n}\right)} \bar{I} \leq \inf _{\mathcal{A}} J_{\mu_{n}}$, using the fact that $\left(M_{1}\left[\mu_{n}\right]\right)$ converges to $M_{1}[\mu]$, and Lemma 2.2 (i) we have that $\limsup _{n \rightarrow+\infty}\left(\sup _{\Gamma\left(\mu_{n}\right)} \bar{I}\right)<+\infty$. Let $\left(n_{j}\right)$ be such that

$$
\limsup _{n \rightarrow+\infty}\left(\sup _{\Gamma\left(\mu_{n}\right)} \bar{I}\right)=\lim _{j \rightarrow+\infty}\left(\sup _{\Gamma\left(\mu_{n_{j}}\right)} \bar{I}\right)
$$

Choose $e_{1}$ a real number independent of $j$, smaller than $\sup _{\Gamma\left(\mu_{n_{j}}\right)} \bar{I}$ for all $j \in \mathbf{N}$ and let $\gamma_{n_{j}} \in \Gamma\left(\mu_{n_{j}}\right)$ be such that

$$
\sup _{\Gamma\left(\mu_{n_{j}}\right)} \bar{I} \leq \bar{I}\left[\gamma_{n_{j}}\right]+1 / n_{j}
$$

One can readily check that $\int_{C} h(\alpha) d \gamma_{n_{j}}$ is less than or equal to $R_{o} M_{1}\left[\mu_{n_{j}}\right]+1-e_{1}$, and so there exists a constant $e_{2}$ independent of $j$ such that

$$
\int_{C}|h(\alpha)| d \gamma_{n_{j}} \leq e_{2}
$$

for all $j \in \mathbf{N}$. By Proposition B.1, (52) implies that there exists a subsequence of $\left(n_{j}\right)$ that we still label $\left(n_{j}\right)$ and a Borel measure $\gamma \in \Gamma(\mu)$ such that $\left(\gamma_{n_{j}}\right)$ converges weak $*$ to $\gamma$. Because $h$ satisfies (4), $\bar{\Lambda}$ is contained in $B_{R_{o}}$ and $\gamma_{n_{j}}\left[(0,+\infty) \times \Lambda^{c} \times \mathbf{R}^{d}\right]=0$ we deduce that there exists a constant $e_{3}$ such that $m_{R}:(\alpha, \mathbf{y}, \mathbf{z}) \rightarrow h(\alpha)-\mathbf{y} \cdot \mathbf{z}-e_{3}+R_{o}|\mathbf{z}|$ is nonnegative for $\gamma_{n_{j}}$-almost every $(\alpha, \mathbf{y}, \mathbf{z}) \in C$. Hence, if we define $k_{R}:(\alpha, \mathbf{y}, \mathbf{z}) \rightarrow$ $l_{R}(\alpha+|\mathbf{y}|+|\mathbf{z}|)$, then

$$
\begin{aligned}
\lim _{j \rightarrow+\infty} \int_{C} m_{R} d \gamma_{n_{j}} & \geq \lim _{j \rightarrow+\infty} \int_{C} m_{R} k_{R} d \gamma_{n_{j}} \\
& =\int_{C} m_{R} k_{R} d \gamma .
\end{aligned}
$$

Consequently,

$$
\lim _{j \rightarrow+\infty} \int_{C}(h(\alpha)-\mathbf{y} \cdot \mathbf{z}) d \gamma_{n_{j}}+R_{o} M_{1}\left[\mu_{n_{j}}\right] \geq \int_{C}(h(\alpha)-\mathbf{y} \cdot \mathbf{z}) k_{R} d \gamma+R_{o} M_{1}[\mu] .
$$

Letting $R$ go to $+\infty$ in $(54)$, using that $\left(M_{1}\left[\mu_{n_{j}}\right]\right)$ converges to $M_{1}[\mu]$ we obtain that

$$
\limsup _{n \rightarrow+\infty}\left(\sup _{\Gamma\left(\mu_{n}\right)} \bar{I}\right) \leq \bar{I}[\gamma] \leq \sup _{\Gamma(\mu)} \bar{I}
$$

and conclude the proof of (ii).

Step 7. Setting $\mu_{n}=\mu$ for all $n \in \mathbf{N}$ in (55) we obtain (iii). 
Theorem 2.1 (duality). Suppose that $h$ satisfies (3), (4), (5) and that $\mu$ satisfies (20). Then the following hold:

(i) There exists a pair $\left(\psi_{\mu}, \phi_{\mu}\right)$ of convex functions minimizing $J_{\mu}$ over $\mathcal{A}$ such that $\left(\psi_{\mu}\right)_{\sharp}=\phi_{\mu}$ and $\left(\phi_{\mu}\right)^{\sharp}=\psi_{\mu}$ and $\operatorname{Lip}\left(\psi_{\mu}\right) \leq R_{o}$. by

(ii) The duality relation $\sup _{\Gamma(\mu)} \bar{I}=\inf _{\mathcal{A}} J_{\mu}$ holds. Defining on $C$ the measure $\gamma$

$$
\int_{C} g d \gamma=\int_{\Lambda} \frac{1}{\beta_{\mu}(\mathbf{y})} g\left(\beta_{\mu}(\mathbf{y}), \mathbf{y}, D \psi_{\mu}^{*}(\mathbf{y})\right) d \mathbf{y}
$$

for all $g \in C_{o}\left(\mathbf{R} \times \mathbf{R}^{d} \times \mathbf{R}^{d}\right)$, we have that $\gamma$ is the unique maximizer of $\bar{I}$ over $\Gamma(\mu)$. Here $\beta_{\mu}: \Lambda \rightarrow(0,+\infty)$ is a Borel map such that $\beta_{\mu}(\mathbf{y})\left(\psi_{\mu}\right)_{\sharp}(\mathbf{y})+\psi_{\mu}\left(D \psi_{\mu}^{*}(\mathbf{y})\right)=$ $\mathbf{y} \cdot D \psi_{\mu}^{*}(\mathbf{y})-h\left(\beta_{\mu}(\mathbf{y})\right)$ for almost every $\mathbf{y} \in \Lambda$.

(iii) If we assume in addition that $\mu[N]=0$ for every $(d-1)$-rectifiable subset $N$ of $\mathbf{R}^{d}$, then $\gamma$ is of the form $\gamma=\gamma^{D \psi}$, i.e., $\gamma$ can be parametrized on $\left(\mathbf{R}^{d}, \mu\right)$ :

$$
\int_{C} g d \gamma=\int_{\mathbf{R}^{d}} g\left(\beta_{\mu}\left(D \psi_{\mu}(\mathbf{z})\right), D \psi_{\mu}(\mathbf{z}), \mathbf{z}\right) d \mu(\mathbf{z})
$$

for all $g \in C_{o}\left(\mathbf{R} \times \mathbf{R}^{d} \times \mathbf{R}^{d}\right)$.

Proof. By Proposition 2.3 there exists a pair $\left(\psi_{\mu}, \phi_{\mu}\right)$ minimizing $J$ over $\mathcal{A}$. By Lemma A.1 (iii)-(iv) the pairs $\left(\psi_{\mu},\left(\psi_{\mu}\right)_{\sharp}\right)$ and $\left(\left(\left(\psi_{\mu}\right)_{\sharp}\right)^{\sharp},\left(\psi_{\mu}\right)_{\sharp}\right)$ minimize $J$ over $\mathcal{A}$ and $\left(\left(\left(\psi_{\mu}\right)_{\sharp}\right)^{\sharp}\right)_{\sharp}=\left(\psi_{\mu}\right)_{\sharp}$. Hence, we may assume without loss of generality that $\psi_{\mu}$, $\phi_{\mu}$ are convex, $\left(\psi_{\mu}\right)_{\sharp}=\phi_{\mu}$, and $\left(\phi_{\mu}\right)^{\sharp}=\psi_{\mu}$, and so

$$
\operatorname{Lip}\left(\psi_{\mu}\right) \leq R_{o}
$$

(see Lemma A.1 ). This concludes the proof of (i).

Step 1 . We first give the proof of (ii) in the special case when there exists $R>0$ such that the support of $\mu$ is contained in $B_{R}$ and $\mu[N]=0$ for every $(d-1)$-rectifiable subset $N$ of $\mathbf{R}^{d}$.

Step 2. For $G \in C_{o}\left(\mathbf{R}^{d}\right)$ and $r>0$ define

$$
\psi_{r}(\mathbf{z}):=\left\{\begin{array}{ccc}
\psi_{\mu}(\mathbf{z})+r G(\mathbf{z}) & \text { if } & \mathbf{z} \in B_{R} \\
+\infty & \text { if } & \mathbf{z} \notin B_{R}
\end{array}\right.
$$

and

$$
\phi_{r}:=\left(\psi_{r}\right)_{\sharp} .
$$

We have that $\psi_{r}^{*}$ is finite at every point of $\mathbf{R}^{d}$ and so $D \psi_{r}^{*}$ exists except on a $(d-$ 1)-rectifiable set (see [1]). Hence, $S_{r}:=D \psi_{r}^{*}: \Lambda \rightarrow B_{R}$ is well-defined $\mu$-almost everywhere. In light of Lemma A.1 let $\beta_{r}: \Lambda \rightarrow(0,+\infty)$ be the unique Borel function such that

$$
\beta_{r}(\mathbf{y}) \phi_{r}(\mathbf{y})+\psi_{r}\left(S_{r}(\mathbf{y})\right)=\mathbf{y} \cdot S_{r}(\mathbf{y})-h\left(\beta_{r}(\mathbf{y})\right) .
$$

Note that $\beta_{r}$ is well-defined $\mu$-almost everywhere. By $(56)\left|\psi_{r}\right|_{L^{\infty}\left(B_{R}\right)}$ is bounded independently of $|r| \leq 1$ and so Lemma A.1 implies

$$
c \leq \beta_{r}(\mathbf{y}) \leq 1 / c
$$


for all $\mathbf{y} \in \Lambda$ and for some constant $c>0$ independent of $r$. Observe that (57) implies

$$
-\frac{r}{\beta_{o}(\mathbf{y})} G\left(S_{o}(\mathbf{y})\right) \leq \phi_{r}(\mathbf{y})-\phi_{o}(\mathbf{y}) \leq-\frac{r}{\beta_{r}(\mathbf{y})} G\left(S_{r}(\mathbf{y})\right)
$$

for all $\mathbf{y} \in \Lambda$. This, together with (58), yields

$$
\left|\phi_{r}(\mathbf{y})-\phi_{o}(\mathbf{y})\right| \leq \frac{r}{c}|G|_{L^{\infty}\left(\mathbf{R}^{d}\right)}
$$

for all $\mathbf{y} \in \Lambda$.

Step 3. Claim. Whenever $S_{o}(\mathbf{y})$ exists we have that $\left(\phi_{r}(\mathbf{y})-\phi_{o}(\mathbf{y})\right) / r$ tends to $-G\left(S_{o}(\mathbf{y})\right) / \beta_{o}(\mathbf{y})$ as $r$ tends to 0 .

Proof. Fix y such that $S_{o}(\mathbf{y})$ exists and assume that $\left(r_{j}\right) \subset(0,+\infty)$ is a sequence converging to 0 ,

$$
S_{r_{j}}(\mathbf{y}) \rightarrow \mathbf{z}_{o}, \quad \beta_{r_{j}}(\mathbf{y}) \rightarrow \alpha_{o},
$$

as $j$ tends to $+\infty$. Since $\left(\psi_{r}\right)$ converges uniformly to $\psi_{o}$ on $B_{R}$ and by $(60)\left(\phi_{r}\right)$ converges uniformly to $\phi_{o}$ on $\Lambda,(57)$ implies that

$$
\alpha_{o} \phi_{o}(\mathbf{y})+\psi_{o}\left(\mathbf{z}_{o}\right)=\mathbf{y} \cdot \mathbf{z}_{o}-h\left(\alpha_{o}\right) .
$$

Since $S_{o}(\mathbf{y})=D \psi_{o}^{*}(\mathbf{y})$ exists, (62) and Lemma A.1 imply

$$
\alpha_{o}=\beta_{o}(\mathbf{y}) \text { and } \mathbf{z}_{o}=S_{o}(\mathbf{y}) .
$$

Because $\left(r_{j}\right) \subset(0,+\infty)$ is arbitrary we deduce that $\left(S_{r}(\mathbf{y})\right)$ converges to $S_{o}(\mathbf{y})$ and $\left(\beta_{r}(\mathbf{y})\right)$ converges to $\beta_{o}(\mathbf{y})$ as $r$ tends to 0 . This together with (59) yields Claim 3.

Step 4. Claim. $S_{o}$ pushes $d \mathbf{y} / \beta_{o}(\mathbf{y})$ forward to $\mu$.

Proof. Note that $J_{\mu}\left[\psi_{o}, \phi_{o}\right]=J_{\mu}\left[\psi_{\mu}, \phi_{\mu}\right]$ and so $\left(\psi_{o}, \phi_{o}\right)$ also minimizes $J_{\mu}$ over $\mathcal{A}$. This combined with Claim 3 implies

$$
0=\lim _{r \rightarrow 0} \frac{J_{\mu}\left[\psi_{r}, \phi_{r}\right]-J_{\mu}\left[\psi_{o}, \phi_{o}\right]}{r}=\int_{\mathbf{R}^{d}} G d \mu-\int_{\Lambda} \frac{G \circ S_{o}}{\beta_{o}} d \mathbf{y} .
$$

Since $G$ is arbitrary in (63), we conclude Claim 4.

Step 5. Using (57) and Claim 4 we have that

$$
\begin{aligned}
J_{\mu}\left[\psi_{o}, \phi_{o}\right]=\int_{\Lambda} \frac{\psi_{o} \circ S_{o}+\beta_{o} \phi_{o}}{\beta_{o}} d \mathbf{y} & =\int_{\Lambda} \frac{\mathbf{y} \cdot S_{o}(\mathbf{y})-h\left(\beta_{o}(\mathbf{y})\right)}{\beta_{o}(\mathbf{y})} d \mathbf{y} \\
& =\int_{C}(\mathbf{y} \cdot \mathbf{z}-h(\alpha)) d \gamma_{\mu}=\bar{I}\left[\gamma_{\mu}\right],
\end{aligned}
$$

where we have defined the measure $\gamma_{\mu}$ by

$$
\int_{C} g d \gamma_{\mu}=\int_{\Lambda} \frac{1}{\beta_{o}(\mathbf{y})} g\left(\beta_{o}(\mathbf{y}), \mathbf{y}, S_{o}(\mathbf{y})\right) d \mathbf{y}
$$

for all $g \in C_{o}\left(\mathbf{R} \times \mathbf{R}^{d} \times \mathbf{R}^{d}\right)$. Clearly $\gamma_{\mu} \in \Gamma(\mu)$. Combining (21) and (64) we deduce that

$$
\sup _{\Gamma(\mu)} \bar{I}=\inf _{\mathcal{A}} J_{\mu}
$$


Step 6. We complete the proof of (ii). Assume now that $\mu$ satisfies only (20). Let $\left(\mu_{n}\right)$ be a sequence of Borel measures on $\mathbf{R}^{d}$ such that $\mu_{n}[N]=0$ whenever $N$ is a $(d-1)$-rectifiable subset of $\mathbf{R}^{d}, M_{o}\left[\mu_{n}\right]=M_{o}[\mu], \operatorname{spt}\left(\mu_{n}\right)$ is bounded for all $n=1,2, \ldots$, and $\left(M_{1}\left[\mu_{n}\right]\right)$ converges to $M_{1}[\mu]$ as $n$ tends to $+\infty$. Combining Proposition 2.3 and (65) we have that

$$
\inf _{\mathcal{A}} J_{\mu} \leq \liminf _{n \rightarrow+\infty}\left(\inf _{\mathcal{A}} J_{\mu_{n}}\right) \leq \limsup _{n \rightarrow+\infty}\left(\sup _{\Gamma\left(\mu_{n}\right)} \bar{I}\right) \leq \sup _{\Gamma(\mu)} \bar{I} .
$$

Combining (21) and (66) we deduce that

$$
\sup _{\Gamma(\mu)} \bar{I}=\inf _{\mathcal{A}} J_{\mu} .
$$

This proves that duality persists under the sole assumption that $\mu$ satisfies only (20). In light of Proposition 2.3 and the above duality result, if $\gamma$ maximizes $\bar{I}$ over $\Gamma(\mu)$, we have that

$$
\int_{C}\left(\psi_{\mu}(\mathbf{z})+\alpha \phi_{\mu}(\mathbf{y})+h(\alpha)-\mathbf{y} \cdot \mathbf{z}\right) d \gamma=0
$$

and so

$$
\psi_{\mu}(\mathbf{z})+\alpha \phi_{\mu}(\mathbf{y})+h(\alpha)-\mathbf{y} \cdot \mathbf{z}=0
$$

for every $(\alpha, \mathbf{y}, \mathbf{z}) \in D^{\prime}$ where $D^{\prime} \subset C$ is such that $\gamma\left[C \backslash D^{\prime}\right]=0$. Let $A$ be the subset of $\Lambda$ where $D \psi_{\mu}^{*}$ exists. Since $H^{d}[\Lambda \backslash A]=0$ we deduce that $\gamma\left[C \backslash D^{\prime \prime}=0\right.$ where

$$
D^{\prime \prime}:=(0,+\infty) \times A \times \mathbf{R}^{d} .
$$

In light of Lemma A.1, there exists a Borel function $\beta_{\mu}: \Lambda \rightarrow(0,+\infty)$ such that

$$
D:=D^{\prime} \cap D^{\prime \prime} \subset\left\{\left(\beta_{\mu}(\mathbf{y}), \mathbf{y}, D \psi_{\mu}^{*}(\mathbf{y})\right) \mid \mathbf{y} \in A\right\} .
$$

Since $\gamma[C \backslash D]=0,(67)$ implies the representation formula

$$
\int_{C} g d \gamma=\int_{\Lambda} \frac{1}{\beta_{\mu}(\mathbf{y})} g\left(\beta_{\mu}(\mathbf{y}), \mathbf{y}, D \psi_{\mu}^{*}(\mathbf{y})\right) d \mathbf{y}
$$

for all $g \in C_{o}\left(\mathbf{R} \times \mathbf{R}^{d} \times \mathbf{R}^{d}\right)$, and so $\gamma$ is uniquely determined. This concludes the proof of (ii).

Step 7. We complete the proof of (iii). Assume that $\mu$ satisfies (20) and $\mu[N]=0$ whenever $N$ is a $(d-1)$-rectifiable subset of $\mathbf{R}^{d}$. Since $\gamma[C]$ is finite, (68) implies that $1 / \beta_{\mu} \in L^{1}(\Lambda)$. Choosing $g \equiv g(\mathbf{z})$ in (68) we obtain that $D \psi_{\mu}^{*}$ is the optimal map in the Monge problem that pushes $d \mathbf{y} / \beta_{\mu}(\mathbf{y})$ forward to $\mu$, and so $D \psi_{\mu}^{*}$ is one-to-one with respect to Lebesgue measure, its inverse is $D \psi_{\mu}$ and is one-to-one with respect to $\mu$ (see Proposition D.1). This together with the representation formula of $\gamma$ given in (ii) proves (iii).

Remark 2.4. Note that if $h$ satisfies (3), (4), (5) and $\mu$ is a measure whose support is contained in $B_{R}$ for some $R>0$, then by Step 1 of the proof of Theorem 2.1 we obtain that $\psi_{\mu}^{*}$ can be extended to a convex, lower semicontinuous function which is finite on $\mathbf{R}^{d}$. If $\beta_{\mu}: \Lambda \rightarrow(0,+\infty)$ is the Borel function such that $\beta_{\mu}(\mathbf{y})\left(\psi_{\mu}\right)_{\sharp}(\mathbf{y})+$ $\psi_{\mu}\left(D \psi_{\mu}^{*}(\mathbf{y})\right)=\mathbf{y} \cdot D \psi_{\mu}^{*}(\mathbf{y})-h\left(\beta_{\mu}(\mathbf{y})\right)$ for almost every $\mathbf{y} \in \Lambda$, and $\psi_{\mu}$ is convex, lower semicontinuous, since $H \circ \beta_{\mu}=\psi_{\mu}^{*}$, we then deduce that there exists a constant $c>0$ such that $c \leq \beta_{\mu} \leq 1 / c$. 
3. Existence of equilibrium configuration. Throughout this section we assume that $\Omega, \Lambda \subset \mathbf{R}^{d}$ are two open bounded sets whose closures are contained in the closed ball $B_{R_{o}}$ of center 0 and radius $R_{o}$. We assume that $h$ satisfies (3), (4), (5) and $\mathbf{F} \in L^{1}(\Omega)^{d}$ is a Borel map. The aim of this section is to prove that a direct consequence of section 2 is that problem

$$
\inf _{(\psi, \phi) \in \mathcal{A}} J[\psi, \phi]
$$

and problem

$$
\sup _{\mathbf{u} \in \mathcal{U}_{\Lambda}^{\prime}} I[\mathbf{u}]
$$

are dual of each other. Here

$$
I[\mathbf{u}]:=\int_{\Omega}(\mathbf{F} \cdot \mathbf{u}-h(|\operatorname{det} D \mathbf{u}|)) d \mathbf{x} \quad\left(\mathbf{u} \in \mathcal{U}_{\Lambda}^{\prime}\right),
$$

and $J$ is defined as in (14) by

$$
J[\psi, \phi]:=\int_{\Omega} \psi(\mathbf{F}(\mathbf{x})) d \mathbf{x}+\int_{\Lambda} \phi(\mathbf{y}) d \mathbf{y} .
$$

We also show that if in addition $\mathbf{F}$ is one-to-one almost everywhere and $\left|\mathbf{F}^{-1}(N)\right|=0$ whenever $N$ is $(d-1)$-rectifiable, then (70) admits a unique minimizer. The inequality

$$
\sup _{\mathbf{u} \in \mathcal{U}_{\Lambda}^{\prime}} I[\mathbf{u}] \leq \inf _{(\psi, \phi) \in \mathcal{A}} J[\psi, \phi]
$$

is straightforward. Indeed, if $\mathbf{u} \in \mathcal{U}_{\Lambda}^{\prime}$ and $(\psi, \phi) \in \mathcal{A}$, then

$$
\mathbf{F} \cdot \mathbf{u}-h(|\operatorname{det} D \mathbf{u}|) \leq \psi \circ \mathbf{F}+|\operatorname{det} D \mathbf{u}| \cdot \phi \circ \mathbf{u}
$$

almost everywhere in $\Omega$, which by integration yields $I[\mathbf{u}] \leq J[\psi, \phi]$. Because $\mathbf{u} \in \mathcal{U}_{\Lambda}^{\prime}$ and $(\psi, \phi) \in \mathcal{A}$ are arbitrary we have that

$$
\sup _{\mathbf{u} \in \mathcal{U}_{\Lambda}^{\prime}} I[\mathbf{u}] \leq \inf _{(\psi, \phi) \in \mathcal{A}} J[\psi, \phi] .
$$

The task in this section is to establish the reverse inequality.

Lemma 3.1. Suppose that (3), (4), and (5) hold and that $\psi_{o}: \mathbf{R}^{d} \rightarrow \mathbf{R}$ is convex, lower semicontinuous. If $\overline{\mathbf{u}} \in \mathcal{U}_{\Lambda}^{\prime}, \mathbf{F}=D \psi_{o}^{*} \circ \overline{\mathbf{u}}$, and $H(|\operatorname{det} D \overline{\mathbf{u}}|)=\left(\psi_{o}\right)^{*} \circ \overline{\mathbf{u}}$, then $I[\overline{\mathbf{u}}]=J\left[\psi_{o},\left(\psi_{o}\right)_{\sharp}\right], \overline{\mathbf{u}}$ is a maximizer of I over $\mathcal{U}_{\Lambda}^{\prime}$, and the pair $\left(\psi_{o},\left(\psi_{o}\right)_{\sharp}\right)$ minimizes $J$ over $\mathcal{A}$.

Proof. Define $\phi_{o}:=\left(\psi_{o}\right)_{\sharp}$. Because $|\operatorname{det} D \overline{\mathbf{u}}| \neq 0$ almost everwhere in the weak sense, we have that $\left|\overline{\mathbf{u}}^{-1}[N]\right|=0$ whenever $|N|=0$. Also, since the convex functions $\phi_{o}$ and $\left(\psi_{o}\right)^{*}$ are differentiable everywhere except on a $(d-1)$-rectifiable set, we have that both $\phi_{o}$ and $\left(\psi_{o}\right)^{*}$ are differentiable at $\overline{\mathbf{u}}(\mathbf{x})$ for almost every $\mathbf{x} \in \Omega$. By Lemma A.1, for these $\mathbf{x} \in \Omega$ we may define $\alpha(\mathbf{x})>0$ and $\mathbf{z}(\mathbf{x}) \in \partial \psi_{o}^{*}(\overline{\mathbf{u}}(\mathbf{x}))$ such that

$$
H(\alpha(\mathbf{x}))=\psi_{o}^{*}(\overline{\mathbf{u}}(\mathbf{x}))
$$

and

$$
\alpha(\mathbf{x}) \phi_{o}(\overline{\mathbf{u}}(\mathbf{x}))+\psi_{o}(\mathbf{z}(\mathbf{x}))=\mathbf{z}(\mathbf{x}) \cdot \overline{\mathbf{u}}(\mathbf{x})-h(\alpha(\mathbf{x})) .
$$


We use the fact that $H$ is decreasing, $H(|\operatorname{det} D \overline{\mathbf{u}}|)=\psi_{o}^{*} \circ \overline{\mathbf{u}}$, and (72) to obtain that

$$
\alpha(\mathbf{x})=|\operatorname{det} D \overline{\mathbf{u}}(\mathbf{x})| .
$$

Since $\psi_{o}^{*}$ is differentiable at $\overline{\mathbf{u}}(\mathbf{x})$ and $\mathbf{z}(\mathbf{x}) \in \partial \psi_{o}^{*}(\overline{\mathbf{u}}(\mathbf{x}))$ we deduce that

$$
\mathbf{z}(\mathbf{x})=\mathbf{F}(\mathbf{x})
$$

By (73), (74), and (75) we obtain that

$$
|\operatorname{det} D \overline{\mathbf{u}}(\mathbf{x})| \phi_{o}(\overline{\mathbf{u}}(\mathbf{x}))+\psi_{o}(\mathbf{F}(\mathbf{x}))=\mathbf{F}(\mathbf{x}) \cdot \overline{\mathbf{u}}(\mathbf{x})-h(|\operatorname{det} D \overline{\mathbf{u}}(\mathbf{x})|),
$$

which by integration yields $I[\overline{\mathbf{u}}]=J\left[\psi_{o}, \phi_{o}\right]$. Since $\left(\psi_{o}, \phi_{o}\right) \in \mathcal{A}(71)$ implies $\overline{\mathbf{u}}$ maximizes $I$ over $\mathcal{U}_{\Lambda}^{\prime}$ and $\left(\psi_{o},\left(\psi_{o}\right)_{\sharp}\right)$ minimizes $J$ over $\mathcal{A}$.

Theorem 3.1 (main results). Suppose that (3), (4), and (5) hold. Then we have the following.

(i) $\inf _{\mathcal{A}} J[\psi, \phi]=\sup _{\mathcal{U}_{\Lambda}^{\prime}} I[\mathbf{u}]$.

(ii) If $\mathbf{F}$ is one-to-one almost everywhere and $(d-1)$-nondegenerate, then I admits a unique maximizer $\overline{\mathbf{u}}$ over $\mathcal{U}_{\Lambda}^{\prime}, \overline{\mathbf{u}}=D \psi_{\mu} \circ \mathbf{F}$, and $I[\overline{\mathbf{u}}]=J\left[\psi_{\mu},\left(\psi_{\mu}\right)_{\sharp}\right]$, and the map $\overline{\mathbf{u}}$ satisfies the Hamilton-Jacobi equation $H(|\operatorname{det} D \overline{\mathbf{u}}|)=\psi_{\mu}^{*} \circ \overline{\mathbf{u}}$ for some lower semicontinuous convex function $\psi_{\mu}: \mathbf{R}^{d} \rightarrow \mathbf{R}$ such that $\operatorname{Lip}\left(\psi_{\mu}\right) \leq R_{o}$ and $\psi_{\mu}=$ $\left(\left(\psi_{\mu}\right)_{\sharp}\right)^{\sharp}$.

(iii) If $\mathbf{F}$ satisfies the assumptions in (ii) and in addition $\mathbf{F} \in L^{\infty}(\Omega)^{d}$, then there exists a constant $c>0$ such that $c \leq|\operatorname{det} D \overline{\mathbf{u}}| \leq 1 / c$, and we may extend $\psi_{\mu}^{*}$ into a Lipschitz, convex function in a neighborhood of conv $(\bar{\Lambda})$.

Proof. We define on $\mathbf{R}^{d}$ the measure $\mu$ given by

$$
\mu[A]:=\left|\mathbf{F}^{-1}[A]\right|
$$

for $A \subset \mathbf{R}^{d}$. Note that

$$
J[\psi, \phi]=\int_{\mathbf{R}^{d}} \psi d \mu+\int_{\Lambda} \phi d \mathbf{y}
$$

which, using the notation of section 2 , is $J_{\mu}[\psi, \phi]$, and the following condition on the moments is satisfied:

$$
M_{o}[\mu]=|\Omega|<+\infty, \quad M_{1}[\mu]=|\mathbf{F}|_{L^{1}(\Omega)}<+\infty .
$$

By Theorem 2.1 (i) there exists a pair $\left(\psi_{\mu}, \phi_{\mu}\right)$ of convex functions minimizing $J_{\mu}$ over $\mathcal{A}$ such that $\left(\psi_{\mu}\right)_{\sharp}=\phi_{\mu}$ and $\left(\phi_{\mu}\right)^{\sharp}=\psi_{\mu}$ and $\operatorname{Lip}\left(\psi_{\mu}\right) \leq R_{o}$.

Step 1. Assume first that $\mathbf{F}$ is one-to-one almost everywhere, $(d-1)$-nondegenerate. Note that $\mu[N]=0$ whenever $N$ is a $(d-1)$-rectifiable subset of $\mathbf{R}^{d}$. Since $\psi_{\mu}$ is convex, the set where $\psi_{\mu}$ is not differentiable is $(d-1)$-rectifiable (see [1]) and so

$$
\overline{\mathbf{u}}(\mathbf{x}):=D \psi_{\mu}(\mathbf{F}(\mathbf{x}))
$$

is defined for almost every $\mathbf{x} \in \Omega$. In light of Theorem 2.1 (iii) $D \psi_{\mu}$ is the optimal map in the Monge problem that pushes $\mu$ forward to $d \mathbf{y} / \beta_{\mu}(\mathbf{y})$ where $\beta_{\mu}: \Lambda \rightarrow(0,+\infty)$ is a Borel function such that

$$
\beta_{\mu}(\mathbf{y})\left(\psi_{\mu}\right)_{\sharp}(\mathbf{y})+\psi_{\mu}\left(D \psi_{\mu}^{*}(\mathbf{y})\right)=\mathbf{y} \cdot D \psi_{\mu}^{*}(\mathbf{y})-h\left(\beta_{\mu}(\mathbf{y})\right)
$$


for almost every $\mathbf{y} \in \Lambda$. Note that in light of Remark 2.4, if in addition $\mathbf{F} \in L^{\infty}(\Omega)^{d}$, then we may assume without loss of generality that $\psi_{\mu}^{*}$ is Lipschitz on $\operatorname{conv}(\bar{\Lambda})$. We have that $D \psi_{\mu}$ is one-to-one on $\mathbf{R}^{d}$ up to a set of zero measure with respect to $\mu$ and $D \psi_{\mu}$ maps $\mathbf{R}^{d}$ onto $\Lambda$. We deduce that

$$
\overline{\mathbf{u}} \text { is one-to-one up to a set of zero measure with respect to } \chi_{\Omega} d \mathbf{x} \text {. }
$$

Recall that in light of Theorem 2.1 (iii) the measure $\gamma_{\mu}$ defined on $C$ by

$$
\int_{C} g d \gamma_{\mu}=\int_{\mathbf{R}^{d}} g\left(\beta_{\mu}\left(D \psi_{\mu}(\mathbf{z})\right), D \psi_{\mu}(\mathbf{z}), \mathbf{z}\right) d \mu(\mathbf{z})
$$

for all $g \in C_{o}\left(\mathbf{R} \times \mathbf{R}^{d} \times \mathbf{R}^{d}\right)$ maximizes $\bar{I}$ over $\Gamma(\mu)$. Therefore we have that

$$
\int_{\Omega} f(\overline{\mathbf{u}}(\mathbf{x})) \beta_{\mu}(\overline{\mathbf{u}}(\mathbf{x})) d \mathbf{x}=\int_{\Lambda} f(\mathbf{y}) d \mathbf{y}
$$

for all $f \in C_{o}\left(\mathbf{R}^{d}\right)$. Consequently,

$$
|\operatorname{det} D \overline{\mathbf{u}}|=\beta_{\mu} \circ \overline{\mathbf{u}} .
$$

Using (78), (79), and the fact that $\beta_{\mu}>0$ we obtain that

$$
\overline{\mathbf{u}} \in \mathcal{U}_{\Lambda}^{\prime}
$$

Since $\beta_{\mu}\left(\psi_{\mu}\right)_{\sharp}+\psi_{\mu} \circ D \psi_{\mu}^{*}=\mathbf{i d} \cdot D \psi_{\mu}^{*}-h \circ \beta_{\mu}$ Lemma A.1 implies $H \circ \beta_{\mu}=\psi_{\mu}^{*}$, and so using (79) we obtain that

$$
H(|\operatorname{det} D \overline{\mathbf{u}}|)=\psi_{\mu}^{*} \circ \overline{\mathbf{u}} .
$$

By Lemma 3.1, (77), (80), and (81) we obtain that $\overline{\mathbf{u}}$ maximizes $I$ over $\mathcal{U}_{\Lambda}^{\prime}$ and $I[\overline{\mathbf{u}}]=J\left[\psi_{\mu},\left(\psi_{\mu}\right)_{\sharp}\right]$. Therefore, we have proved (i) under the assumption that $\mathbf{F}$ is one-to-one almost everywhere, $(d-1)$-nondegenerate.

Step 2. We prove that $\overline{\mathbf{u}}$ is the unique maximizer of $I$ over $\mathcal{U}_{\Lambda}^{\prime}$. Indeed, if $\mathbf{u}$ is another maximizer of $I$ over $\mathcal{U}_{\Lambda}^{\prime}$, the duality relation between (10) and (13) implies

$$
\mathbf{F}(\mathbf{x}) \cdot \mathbf{u}(\mathbf{x})-h(|\operatorname{det} D \mathbf{u}(\mathbf{x})|)=\psi_{\mu}(\mathbf{F}(\mathbf{x}))+|\operatorname{det} D \mathbf{u}(\mathbf{x})| \phi_{\mu}(\mathbf{u}(\mathbf{x}))
$$

for all almost every $\mathbf{x} \in \Omega$, and so, by Lemma A.1 (i),

$$
\mathbf{u}(\mathbf{x}) \in \partial \psi_{\mu}(\mathbf{F}(\mathbf{x}))
$$

for these $\mathbf{x}$. Since $\psi_{\mu}$ is differentiable everywhere in $B_{R}$ except on a $(d-1)$-rectifiable set and $\mathbf{F}$ is $(d-1)$-nondegenerate, (82) implies $\mathbf{u}(\mathbf{x})=D \psi_{\mu}(\mathbf{F}(\mathbf{x}))=\overline{\mathbf{u}}(\mathbf{x})$ for all almost every $\mathbf{x} \in \Omega$. This concludes the proof of (ii).

Step 3. If $\mathbf{F}$ satisfies the assumptions in (ii) and in addition $\mathbf{F} \in L^{\infty}(\Omega)^{d}$, then there exists $R>0$ such that the support of $\mu$ is contained in $B_{R}$. Using Remark 2.4 and (79) we obtain (iii).

Step 4. We now prove (i) under the sole assumption that $\mathbf{F} \in L^{1}(\Omega)^{d}$. For each $n \in \mathbf{N}$ we may find $\mathbf{F}_{n} \in L^{\infty}(\Omega)^{d}$ that is one-to-one almost everywhere, $(d-1)$ nondegenerate, and such that

$$
\left|\mathbf{F}_{n}-\mathbf{F}\right|_{L^{1}(\Omega)} \rightarrow 0
$$


as $n$ tends to $+\infty$. Define

$$
J_{n}[\psi, \phi]:=\int_{\mathbf{R}^{d}} \psi\left(\mathbf{F}_{n}(\mathbf{x})\right) d \mathbf{x}+\int_{\Lambda} \phi(\mathbf{y}) d \mathbf{y}
$$

and

$$
I_{n}[\mathbf{u}]:=\int_{\Omega}\left(\mathbf{F}_{n} \cdot \mathbf{u}-h(|\operatorname{det} D \mathbf{u}|)\right) d \mathbf{x} .
$$

By (ii) there exists $\psi_{n}: \mathbf{R}^{d} \rightarrow \mathbf{R}$ convex function such that $\operatorname{Lip}\left(\psi_{n}\right) \leq R_{o}$ and

$$
J_{n}\left[\psi_{n},\left(\psi_{n}\right)_{\sharp}\right]=\inf _{\mathcal{A}} J_{n}=\sup _{\mathcal{U}_{\Lambda}^{\prime}} I_{n} .
$$

Using that $\operatorname{Lip}\left(\psi_{n}\right) \leq R_{o}$ we have that

$$
J_{n}\left[\psi_{n},\left(\psi_{n}\right)_{\sharp}\right] \geq \inf _{\mathcal{A}} J-R_{o}\left|\mathbf{F}_{n}-\mathbf{F}\right|_{L^{1}(\Omega)}
$$

and using that $\mathbf{u}(\Omega) \subset \Lambda \subset B_{R_{o}}$ for all $\mathbf{u} \in \mathcal{U}_{\Lambda}^{\prime}$ we deduce that

$$
\sup _{\mathcal{U}_{\Lambda}^{\prime}} I_{n} \leq \sup _{\mathcal{U}_{\Lambda}^{\prime}} I+R_{o}\left|\mathbf{F}_{n}-\mathbf{F}\right|_{L^{1}(\Omega)} .
$$

Combining (83), (84), and (85) we obtain (i).

Corollary 3.2 (characterization of maximizers of $I$ ). Suppose that (3), (4), (5) hold and that $\mathbf{F} \in L^{1}(\Omega)^{d}$. Assume that $\overline{\mathbf{u}} \in \mathcal{U}_{\Lambda}^{\prime}$. Then $\overline{\mathbf{u}}$ maximizes $I$ over $\mathcal{U}_{\Lambda}^{\prime}$ if and only if there exists a lower semicontinuous convex function $\psi_{o}: \mathbf{R}^{d} \rightarrow \mathbf{R}$ such that $D \psi_{o}^{*}$ exists almost everywhere in $\Lambda, \mathbf{F}=D \psi_{o}^{*} \circ \overline{\mathbf{u}}$, and $H(|\operatorname{det} D \overline{\mathbf{u}}|)=\psi_{o}^{*} \circ \overline{\mathbf{u}}$ on $\Omega$.

Proof. Step 1. Assume that $\overline{\mathbf{u}}$ maximizes $I$ over $\mathcal{U}_{\Lambda}^{\prime}$. By Theorem 3.1 there exists a lower semicontinuous convex function $\psi_{o}: \mathbf{R}^{d} \rightarrow \mathbf{R}$ such that $I[\overline{\mathbf{u}}]=J\left[\psi_{o}, \phi_{o}\right]$ and $\psi_{o}=\left(\phi_{o}\right)^{\sharp}$, where $\phi_{o}:=\left(\psi_{o}\right)_{\sharp}$. We deduce that

$$
\left.|\operatorname{det} D \overline{\mathbf{u}}(\mathbf{x})| \phi_{o}(\overline{\mathbf{u}}(\mathbf{x}))+\psi_{o}(\mathbf{F}(\mathbf{x}))=\mathbf{F}(\mathbf{x}) \cdot \overline{\mathbf{u}}(\mathbf{x})-h(|\operatorname{det} D \overline{\mathbf{u}}(\mathbf{x})|)\right)
$$

for almost every $\mathbf{x} \in \Omega$. Since $\psi_{o}^{*}$ is differentiable at almost every $\overline{\mathbf{u}}(\mathbf{x})$, using (86) and Lemma A.1 we deduce that

$$
\mathbf{F}=D \psi_{o}^{*} \circ \overline{\mathbf{u}}
$$

and

$$
H(|\operatorname{det} D \overline{\mathbf{u}}|)=\psi_{o}^{*} \circ \overline{\mathbf{u}} .
$$

Step 2. The converse implication is given by Lemma 3.1, and we conclude the proof of the lemma.

4. Smoothness of equilibrium configurations. Throughout this section, unless the contrary is explicitly stated, we assume that $\Omega, \Lambda \subset \mathbf{R}^{d}$ are two open bounded sets. Recall that $d \geq 2$ is an integer. We now state the main result of this section.

THEOREM 4.1 (smoothness of maximizers of $I$ ). Assume that $\Omega$ is connected, its boundary $\partial \Omega$ is Lipschitz, $\Lambda$ and $\mathbf{F}(\bar{\Omega})$ are convex. Assume that $\mathbf{F}$, $\operatorname{det} D \mathbf{F} \in C^{1}(\bar{\Omega})^{d}$, $0<\operatorname{det} D \mathbf{F}$ on $\bar{\Omega}, \mathbf{F}$ is a homeomorphism of $\bar{\Omega}$ onto $\mathbf{F}(\bar{\Omega})$. If $h$ satisfies (3), (4), and (5), then the following hold: 
(i) Problem $\sup _{\mathcal{A}}-J$ and $\inf _{\mathcal{U}_{\Lambda}} E$ are dual to each other, and there exists a unique $\overline{\mathbf{u}}$ minimizing $E$ over $\mathcal{U}_{\Lambda}$.

(ii) We have that $\overline{\mathbf{u}} \in C^{1}(\Omega)^{d} \cap C^{0, s}(\bar{\Omega})^{d}$, $\operatorname{det} D \overline{\mathbf{u}} \in C^{0, s}(\bar{\Omega}) \cap C^{1}(\Omega)$ for all $0<s<1$, and $\operatorname{det} D \overline{\mathbf{u}}+1 / \operatorname{det} D \overline{\mathbf{u}} \in L^{\infty}(\Omega)$.

(iii) Furthermore, $\overline{\mathbf{u}}$ satisfies the partial differential equations (9) in the weak sense and (11) pointwise.

Proof. Step 1. To show (i), it suffices to check that the map $\overline{\mathbf{u}}$ maximizing $I$ over $\mathcal{U}_{\Lambda}^{\prime}$ belongs to $\mathcal{U}_{\Lambda}$. By Theorem 3.1 there exists a lower semicontinuous, convex function $\psi_{o}: \mathbf{R}^{d} \rightarrow \mathbf{R}$ such that $\overline{\mathbf{u}}:=D \psi_{o} \circ \mathbf{F} \in \mathcal{U}_{\Lambda}^{\prime}, H \circ|\operatorname{det} D \overline{\mathbf{u}}|=\psi_{o}^{*} \circ \overline{\mathbf{u}}$,

$$
I[\overline{\mathbf{u}}]=J\left[\psi_{o},\left(\psi_{o}\right)_{\sharp}\right],
$$

and $D \psi_{o}$ pushes $f_{o} d \mathbf{z}$ forward to $d \mathbf{y} / \beta_{o}(\mathbf{y})$, where

$$
f_{o}(\mathbf{z}):=\frac{1}{\operatorname{det} D \mathbf{F}\left(\mathbf{F}^{-1}(\mathbf{z})\right)} \quad(\mathbf{z} \in \mathbf{F}(\bar{\Omega}))
$$

and $\beta_{o}: \Lambda \rightarrow(0,+\infty)$ is defined by $\beta_{o}(\mathbf{y})\left(\psi_{o}\right)_{\sharp}(\mathbf{y})+\psi_{o}\left(D \psi_{o}^{*}(\mathbf{y})\right)=\mathbf{y} \cdot D \psi_{o}^{*}(\mathbf{y})-$ $h\left(\beta_{o}(\mathbf{y})\right)$. By Lemma A.1 we have that

$$
H \circ \beta_{o}=\psi_{o}^{*} .
$$

Since $\mathbf{F}$ is bounded we may assume without loss of generality that $\psi_{o}^{*}$ is Lipschitz on $\bar{\Lambda}$ and because the inverse $H^{-1}$ of $H$ is of class $C^{1}$, (90) and Proposition D.2 imply that $\beta_{o} \in C^{1}(\bar{\Lambda})$. Clearly, $f_{o}$ is of class $C^{1}$, bounded below and above on $\mathbf{F}(\bar{\Omega})$. Using Proposition D.2 again, using that $D \psi_{o}$ pushes $f_{o} d \mathbf{z}$ forward to $d \mathbf{y} / \beta_{o}(\mathbf{y})$ and that the density functions $f_{o}$ and $1 / \beta_{o}(\mathbf{y})$ are smooth we deduce that $D \psi_{o} \in$ $C^{0, s}(\mathbf{F}(\bar{\Omega}))^{d} \cap C^{1, s}(\mathbf{F}(\Omega))^{d}$ for all $0<s<1$. This proves (i) and (ii). Note that there exists a constant $c>0$ such that

$$
c \leq \operatorname{det} D \overline{\mathbf{u}} \leq 1 / c
$$

Step 2. Let $\mathbf{v} \in C_{o}^{\infty}(\Omega)^{d}$, let $K$ be the support of $\mathbf{v}$, and for each $|r|<1$ define

$$
\mathbf{u}_{r}:=\overline{\mathbf{u}}+r \mathbf{v} .
$$

Since $\overline{\mathbf{u}} \in C^{1}(K), \mathbf{u}_{r}=\overline{\mathbf{u}}$ on $\Omega \backslash K$, and (91) holds we deduce that $\left(D \mathbf{u}_{r}\right)$ converges uniformly to $D \overline{\mathbf{u}}$ on $\Omega$ and there exists $r_{o}>0$ such that

$$
c / 2 \leq \operatorname{det} D \mathbf{u}_{r}(\mathbf{x}) \leq 2 / c
$$

for almost every $\mathbf{x} \in \Omega$ and for every $|r|<r_{o}$. Thanks to Remark 4.1, since $\mathbf{u}_{r} \in$ $C^{1}(\Omega)^{d} \cap C(\bar{\Omega})^{d}, \mathbf{u}_{r}$ and $\overline{\mathbf{u}}$ agree on $\partial \Omega$, (92) implies that $\mathbf{u}_{r}$ is one-to-one from $\bar{\Omega}$ onto $\overline{\mathbf{u}}(\bar{\Omega})$ and $\mathbf{u}_{r} \in \mathcal{U}_{\Lambda}$. Using that $\overline{\mathbf{u}}$ maximizes $I$ over $\mathcal{U}_{\Lambda}$ we have that

$$
\text { (93) } 0=-\lim _{r \rightarrow 0}\left(I\left[\mathbf{u}_{r}\right]-I[\overline{\mathbf{u}}]\right) / r=\lim _{r \rightarrow 0} \int_{K}\left(W\left(D \mathbf{u}_{r}\right)-W(D \overline{\mathbf{u}})\right) / r d \mathbf{x}-\int_{K} \mathbf{F} \cdot \mathbf{v} d \mathbf{x} .
$$

Since $\left(D \mathbf{u}_{r}\right)$ converges uniformly to $D \overline{\mathbf{u}}$ on $\Omega,\left\{A d j D \mathbf{u}_{r}\right\}_{r}$ and $\operatorname{Adj} D \overline{\mathbf{u}}$ are uniformly bounded by a constant $c_{1}>0$. Now note that $D W$ is bounded on $\left\{M \in \mathbf{R}^{d \times d}\right.$ : $\left.c / 2 \leq \operatorname{det} M \leq 2 / c,|A d j M|<c_{1}\right\}$, and so (92) and (93) yield

$$
0=\int_{K} D W(D \overline{\mathbf{u}}) \cdot D \mathbf{v} d \mathbf{x}=\int_{K} \mathbf{F} \cdot \mathbf{v} d \mathbf{x} .
$$


Since $\mathbf{v}$ is arbitrary in (94) we read off

$$
-\operatorname{div}(D W(D \overline{\mathbf{u}}))=\mathbf{F} \quad \text { in } \Omega
$$

in the weak sense.

This concludes the proof of Theorem 4.1.

Remark 4.1. If $\mathbf{u}_{o} \in C^{1}(\Omega)^{d} \cap C(\bar{\Omega})^{d}$ is one-to-one on $\Omega$, $\operatorname{det} D \mathbf{u}_{o}$ is positive, and $\mathbf{u}_{o}(\Omega):=\Lambda$, then by the invariance of domain theorem the set $\Lambda$ is open (see [16]). If $\mathbf{u} \in C^{1}(\Omega)^{d} \cap C(\bar{\Omega})^{d}$ agrees with $\mathbf{u}_{o}$ on $\partial \Omega$, then

$$
\operatorname{deg}(\mathbf{u}, \Omega, \mathbf{y})=\operatorname{deg}\left(\mathbf{u}_{o}, \Omega, \mathbf{y}\right)=\left\{\begin{array}{lll}
1 & \text { if } & \mathbf{y} \in \Lambda \\
0 & \text { if } & \mathbf{y} \notin \bar{\Lambda},
\end{array}\right.
$$

where $\operatorname{deg}(\mathbf{u}, \Omega, \mathbf{y})$ stands for the topological degree of $\mathbf{u}$ at $\mathbf{y}$ on $\Omega$. If in addition $\operatorname{det} D \mathbf{u}>0$ in $\Omega$, then (95) implies $\mathbf{u}$ is one-to-one and $\mathbf{u}(\Omega)=\Lambda$. Hence $\mathbf{u} \in \mathcal{U}_{\Lambda}$. In particular, $\mathcal{U}_{o}$ is a subset of $\mathcal{U}_{\Lambda}$ (see, for instance, [16] for properties of the topological degree theory).

Corollary 4.2. Assume that $\mathbf{u}_{o} \in C^{1}(\Omega)^{d} \cap C(\bar{\Omega})^{d}$ is one-to-one on $\bar{\Omega}$, $\operatorname{det} D \mathbf{u}_{o}$ is positive and belongs to $C^{1}(\Omega)$, $\operatorname{det} D \mathbf{u}_{o}+1 / \operatorname{det} D \mathbf{u}_{o} \in L^{\infty}(\Omega)$, and $\mathbf{u}_{o}(\Omega)=\Lambda$. Under the assumptions of Theorem 4.1 the infima in (7) and (8) coincide.

Proof. Thanks to Remark 4.1 we have that $\inf _{\mathcal{U}_{\Lambda}} E \leq \inf _{\mathcal{U}_{o}} E$. To conclude the proof of the corollary it suffices to show the reverse inequality. Let $\overline{\mathbf{u}}$ be the minimizer of $E$ over $\mathcal{U}_{\Lambda}$. By Proposition C.1 there exists a sequence $\left(\mathbf{u}_{n}\right) \subset \mathcal{U}_{o}$ such that $\left\|\mathbf{u}_{n}-\overline{\mathbf{u}}\right\|_{1}\|\mathbf{F}\|_{\infty} \leq 1 / n$ and

$$
\operatorname{det} D \mathbf{u}_{n}=\operatorname{det} D \overline{\mathbf{u}} \quad \text { almost everywhere in } \Omega
$$

for each $n=1,2, \ldots$. We have that

$$
E\left[\mathbf{u}_{n}\right]=E[\overline{\mathbf{u}}]+\int_{\Omega} \mathbf{F} \cdot\left(\overline{\mathbf{u}}-\mathbf{u}_{n}\right) d \mathbf{x} \leq \inf _{\mathcal{U}_{\Lambda}} E+1 / n .
$$

This concludes the proof of Corollary 4.2.

Appendix A. Properties of the map $\phi \rightarrow \phi^{\sharp}$. Throughout this section $\Lambda$ is an open subset of $\mathbf{R}^{d}$ contained in the closed ball $B_{R}$ of center 0 and radius $R>0$, $h \in C^{2}(0,+\infty)$ is strictly convex and satisfies the growth conditions (4). Recall that

$$
H(t):=h(t)-t h^{\prime}(t) \quad(t \in(0,+\infty)) .
$$

Suppose that $\tilde{\phi}: \operatorname{conv}(\Lambda) \rightarrow \mathbf{R}, \tilde{\psi}: \mathbf{R}^{d} \rightarrow \mathbf{R} \cup\{+\infty\}$ are lower semicontinuous, and define the convex functions

$$
\psi(\mathbf{z})=\tilde{\phi}^{\sharp}(\mathbf{z}):=\sup _{\mathbf{y} \in \operatorname{conv}(\Lambda)}\left\{\mathbf{y} \cdot \mathbf{z}+h^{*}(-\tilde{\phi}(\mathbf{y}))\right\} \quad\left(\mathbf{z} \in \mathbf{R}^{d}\right),
$$

and

$$
\phi(\mathbf{y})=\tilde{\psi}_{\sharp}(\mathbf{y}):=\sup _{\alpha>0}\left\{\frac{(\tilde{\psi})^{*}(\mathbf{y})-h(\alpha)}{\alpha}\right\} \quad\left(\mathbf{y} \in \mathbf{R}^{d}\right) .
$$

Lemma A.1. Let $\mathbf{y}_{o}, \mathbf{z}_{o} \in \mathbf{R}^{d}$. The following statements hold:

(i) The supremum in $\phi\left(\mathbf{y}_{o}\right)$ is attained for $\beta\left(\mathbf{y}_{o}\right) \in(0,+\infty)$ provided that $(\tilde{\psi})^{*}\left(\mathbf{y}_{o}\right)$ is finite. If $S\left(\mathbf{y}_{o}\right) \in \partial(\tilde{\psi})^{*}\left(\mathbf{y}_{o}\right)$, then we have that $S\left(\mathbf{y}_{o}\right) \in \beta\left(\mathbf{y}_{o}\right) \partial \phi\left(\mathbf{y}_{o}\right)$, and $H\left(\beta\left(\mathbf{y}_{o}\right)\right)$ 
$=(\tilde{\psi})^{*}\left(\mathbf{y}_{o}\right)$. Consequently, the pair $\left(\beta\left(\mathbf{y}_{o}\right), S\left(\mathbf{y}_{o}\right)\right)$ or in other words the pair $\left(\beta\left(\mathbf{y}_{o}\right)\right.$, $\left.D \tilde{\psi}^{*}\left(\mathbf{y}_{o}\right)\right)$ is uniquely determined if $(\tilde{\psi})^{*}$ is differentiable at $\mathbf{y}_{o} ; \beta, S$ are Borel functions.

(ii) If $\psi \not \equiv+\infty$, then $\operatorname{Lip}(\psi) \leq R$.

(iii) If $(\tilde{\psi}, \tilde{\phi}) \in \mathcal{A}$, then $\phi \leq \tilde{\phi}$ on $\operatorname{conv}(\Lambda)$ and $\psi \leq \tilde{\psi}$ on $\mathbf{R}^{d}$.

(iv) We have that $\left(\left(\tilde{\phi}^{\sharp}\right)_{\sharp}\right)^{\sharp}=\tilde{\phi}^{\sharp}$ on $\mathbf{R}^{d}$ and $\left(\left(\tilde{\psi}_{\sharp}\right)^{\sharp}\right)_{\sharp}=\tilde{\psi}_{\sharp}$ on $\operatorname{conv}(\Lambda)$.

Proof. Step 1. We first prove (i). Note that in light of $(4), \phi\left(\mathbf{y}_{o}\right)$ is finite if and only if $(\tilde{\psi})^{*}(\mathbf{y})$ is finite, in which case existence of a maximizer $\beta\left(\mathbf{y}_{o}\right)$ in $\phi\left(\mathbf{y}_{o}\right)$ is a straightforward to obtain. Next, observe that if $S\left(\mathbf{y}_{o}\right) \in \partial(\tilde{\psi})^{*}\left(\mathbf{y}_{o}\right)$, then the auxiliary function $K:(\alpha, \mathbf{y}, \mathbf{z}) \rightarrow \alpha \phi(\mathbf{y})+\tilde{\psi}(\mathbf{z})+h(\alpha)-\mathbf{y} \cdot \mathbf{z}$ attains its minimum at $\left(\beta\left(\mathbf{y}_{o}\right), \mathbf{y}_{o}, S\left(\mathbf{y}_{o}\right)\right)$. Exploiting the fact that both functions $K$ and $\frac{\partial K}{\partial \alpha}$ vanish at $\left(\beta\left(\mathbf{y}_{o}\right), \mathbf{y}_{o}, S\left(\mathbf{y}_{o}\right)\right)$ we deduce that

$$
-\phi\left(\mathbf{y}_{o}\right)=h^{\prime}\left(\beta\left(\mathbf{y}_{o}\right)\right) \text { and } H\left(\beta\left(\mathbf{y}_{o}\right)\right)=\mathbf{y}_{o} \cdot S\left(\mathbf{y}_{o}\right)-\tilde{\psi}\left(S\left(\mathbf{y}_{o}\right)\right) .
$$

Step 2. Since $K\left(\beta\left(\mathbf{y}_{o}\right), \mathbf{y}_{o}, \mathbf{z}\right)$ and $K\left(\beta\left(\mathbf{y}_{o}\right), \mathbf{y}, S\left(\mathbf{y}_{o}\right)\right)$ are greater than or equal to $K\left(\beta\left(\mathbf{y}_{o}\right), \mathbf{y}_{o}, S\left(\mathbf{y}_{o}\right)\right)$, we readily deduce that $S\left(\mathbf{y}_{o}\right) \in \beta\left(\mathbf{y}_{o}\right) \partial \phi\left(\mathbf{y}_{o}\right)$. Using the fact that $\tilde{\psi}\left(S\left(\mathbf{y}_{o}\right)\right)+(\tilde{\psi})^{*}\left(\mathbf{y}_{o}\right)=\mathbf{y}_{o} \cdot S\left(\mathbf{y}_{o}\right)$, the equation $H\left(\beta\left(\mathbf{y}_{o}\right)\right)=\mathbf{y}_{o} \cdot S\left(\mathbf{y}_{o}\right)-\tilde{\psi}\left(S\left(\mathbf{y}_{o}\right)\right)$ reads off $H\left(\beta\left(\mathbf{y}_{o}\right)\right)=(\tilde{\psi})^{*}\left(\mathbf{y}_{o}\right)$. This concludes the proof of (i). Since $\Lambda \subset B_{R}$ we conclude (ii).

Step 3. The proof of (iii) is straightforward.

Step 4 . We now prove (iv). We have that $\left(\tilde{\phi}^{\sharp},\left(\tilde{\phi}^{\sharp}\right)_{\sharp}\right) \in \mathcal{A}$ and because $\left(\tilde{\phi}^{\sharp}, \tilde{\phi}\right) \in \mathcal{A}$, (iii) implies that $\left(\tilde{\phi}^{\sharp}\right)_{\sharp} \leq \tilde{\phi}$ on $\operatorname{conv}(\Lambda)$. Using the fact that the operator $\varphi \rightarrow \varphi^{\sharp}$ is nonincreasing we deduce that $\left(\left(\tilde{\phi}^{\sharp}\right)_{\sharp}\right)^{\sharp} \geq \tilde{\phi}^{\sharp}$ on $\mathbf{R}^{d}$. But (iii) and $\left(\tilde{\phi}^{\sharp},\left(\tilde{\phi}^{\sharp}\right)_{\sharp}\right) \in \mathcal{A}$ also imply that $\left(\left(\tilde{\phi}^{\sharp}\right)_{\sharp}\right)^{\sharp} \leq \tilde{\phi}^{\sharp}$ on $\mathbf{R}^{d}$. Consequently, $\left(\left(\tilde{\phi}^{\sharp}\right)_{\sharp}\right)^{\sharp}=\tilde{\phi}^{\sharp}$ on $\mathbf{R}^{d}$. Likewise, $\left(\left(\tilde{\psi}_{\sharp}\right)^{\sharp}\right)_{\sharp}=\tilde{\psi}_{\sharp}$ on $\operatorname{conv}(\Lambda)$.

This concludes the proof of Lemma A.1.

Lemma A.2. Suppose that $\tilde{\psi} \equiv+\infty$ on the complement of $B_{R}$ and that $|\tilde{\psi}|_{L^{\infty}\left(B_{R}\right)}$ $<+\infty$. Let $\beta$ be defined as in Lemma A.1. Then there exists a constant $c$ depending only on $h, R$, and $|\tilde{\psi}|_{L^{\infty}\left(B_{R}\right)}$ such that $c \leq \beta(\mathbf{y}) \leq 1 / c$ for all $\mathbf{y}$.

Proof. Set $t_{o}:=R^{2}+|\tilde{\psi}|_{L^{\infty}\left(B_{R}\right)}$. Since $\tilde{\psi} \equiv+\infty$ on the complement of $B_{R}$ we obtain that $\left|(\tilde{\psi})^{*}\right|_{L^{\infty}\left(B_{R}\right)} \leq t_{o}$. Using (12) and Lemma A.1 (i) we conclude the lemma with $c:=\max \left\{H^{-1}\left(t_{o}\right), 1 / H^{-1}\left(-t_{o}\right)\right\}$.

Appendix B. Compacity of a special class of measures. Throughout this section we assume that $\Lambda \subset \mathbf{R}^{d}$ is an open bounded set whose closure is contained in the closed ball $B_{R_{o}}$ of center 0 and radius $R_{o}$. If $\mu$ is a finite positive measure on $\mathbf{R}^{d}$, we recall that the moments $M_{o}(\mu)$ and $M_{o}(\mu)$ are defined in $(20), C:=(0, \infty) \times \mathbf{R}^{d} \times \mathbf{R}^{d}$, and $\Gamma[\mu]$ is the set of all Borel measures on $C$ such that

$$
\int_{C} f(\mathbf{z}) d \gamma(\alpha, \mathbf{y}, \mathbf{z})=\int_{\mathbf{R}^{d}} f(\mathbf{z}) d \mu(\mathbf{z})
$$

and

$$
\int_{C} \alpha f(\mathbf{y}) d \gamma(\alpha, \mathbf{y}, \mathbf{z})=\int_{\Lambda} f(\mathbf{y}) d \mathbf{y}
$$

for all $f \in C_{o}\left(\mathbf{R}^{d}\right)$.

Proposition B.1. Suppose that $\mu$ satisfies $(20)$, that $\left(\mu_{n}\right)$ is a sequence of Borel measures converging weak $*$ to $\mu, M_{o}\left[\mu_{n}\right]=M_{o}[\mu](n=1,2, \ldots)$, and that 
$h$ satisfies (4). If $\gamma_{n} \in \Gamma\left(\mu_{n}\right)$ and the sequence of real numbers $\left(\int_{C}|h(\alpha)| d \gamma_{n}\right)$ is bounded independently of $n$, then there exists a sequence $\left(n_{j}\right) \subset \mathbf{N}$ and a Borel measure $\gamma \in \Gamma(\mu)$ such that $\left(\gamma_{n_{j}}\right)$ converges weak $*$ to $\gamma$.

Proof. Because $\gamma_{n} \in \Gamma\left(\mu_{n}\right)$ we have that $\gamma_{n}[C]=M_{o}[\mu]$, and so there exists a sequence $\left(n_{j}\right) \subset \mathbf{N}$ and a Borel measure $\gamma$ on $C$ such that $\left(\gamma_{n_{j}}\right)$ converges weak $*$ to $\gamma$. We next introduce the functions

$$
k(\alpha, \mathbf{y}):=l_{R}(\alpha+|\mathbf{y}|) \quad\left(\alpha>0, \mathbf{y} \in \mathbf{R}^{d}\right),
$$

where, for $R>1, l_{R}: \mathbf{R} \rightarrow[0,1]$ is of class $C^{\infty}$ and satisfies

$$
l_{R}(t)=\left\{\begin{array}{llc}
1 & \text { if } & |t| \leq R-1 \\
0 & \text { if } & |t| \geq R
\end{array}\right.
$$

If $f \in C_{o}\left(\mathbf{R}^{d}\right)$, then

$$
\begin{aligned}
\left|\int_{C} f(\mathbf{z})(1-k(\alpha, \mathbf{y})) d \gamma_{n_{j}}\right| & =\left|\int_{\alpha>(R-1) / 2} f(\mathbf{z})(1-k(\alpha, \mathbf{y})) d \gamma_{n_{j}}\right| \\
& \leq 2\left(|f|_{\infty}|\Lambda|\right) /(R-1) .
\end{aligned}
$$

Using (99) and the fact that $\gamma_{n_{j}} \in \Gamma\left(\mu_{n_{j}}\right)$ we have that

$$
\left|\int_{\mathbf{R}^{d}} f(\mathbf{z}) d \mu_{n_{j}}(\mathbf{z})-\int_{C} f(\mathbf{z}) k(\alpha, \mathbf{y}) d \gamma_{n_{j}}\right| \leq 2\left(|f|_{\infty}|\Lambda|\right) /(R-1) .
$$

Letting first $j$ go to $+\infty$ and then $R$ go to $+\infty$ in (100) we deduce that

$$
\int_{\mathbf{R}^{d}} f(\mathbf{z}) d \mu(\mathbf{z})=\int_{C} f(\mathbf{z}) d \gamma .
$$

Define the function

$$
\beta(R):=M \sup _{t}\{t /|h(t)| \mid t \geq(R-1) / 2\} \quad(R>1),
$$

where $M>0$ is a constant independent of $n$ such that $\int_{C}|h(\alpha)| d \gamma_{n} \leq M$ for all $n \in \mathbf{N}$. Since $\gamma_{n_{j}} \in \Gamma\left(\mu_{n_{j}}\right)$, if $A_{R}$ is the subset of all $(\alpha, \mathbf{y}, \mathbf{z}) \in C$ such that $|\mathbf{z}|>(R-1) / 2$ and $|\alpha| \leq(R-1) / 2$, then we have that

$$
\left|\int_{\Lambda} f(\mathbf{y}) d \mathbf{y}-\int_{C} \alpha f(\mathbf{y}) k(\alpha, \mathbf{z}) d \gamma_{n_{j}}\right| \leq\left|\int_{C} \alpha f(\mathbf{y})(1-k(\alpha, \mathbf{z})) d \gamma_{n_{j}}\right|
$$

and

$$
\begin{aligned}
\left|\int_{C} \alpha f(\mathbf{y})(1-k(\alpha, \mathbf{z})) d \gamma_{n_{j}}\right| & \leq 2 \int_{\alpha>(R-1) / 2} \alpha|f(\mathbf{y})|(1-k(\alpha, \mathbf{z})) d \gamma_{n_{j}} \\
& +\int_{A_{R}} \alpha|f(\mathbf{y})|(1-k(\alpha, \mathbf{z})) d \gamma_{n_{j}} \\
& \leq 2|f|_{\infty}\left(\beta(R)+R\left(\mu\left[B_{\frac{R-1}{2}}^{c}\right]+1 / n_{j}\right)\right) .
\end{aligned}
$$

Hence

$$
\left|\int_{C} \alpha f(\mathbf{y})(1-k(\alpha, \mathbf{z})) d \gamma_{n_{j}}\right| \leq 2|f|_{\infty}\left(\beta(R)+\int_{B_{\frac{R-1}{2}}^{c}}(2|\mathbf{z}|+1) d \mu+R / n_{j}\right) .
$$


In light of $(4) \beta(R)$ tends to 0 as $R$ tends to $+\infty$. Using (102) and letting first $j$ go to $+\infty$ and then $R$ tend to $+\infty$ in (103), since $M_{o}[\mu], M_{1}[\mu]<+\infty$, we deduce that

$$
\int_{\Lambda} f(\mathbf{y}) d \mathbf{y}=\int_{C} \alpha f(\mathbf{y}) d \gamma
$$

Since $f \in C_{o}\left(\mathbf{R}^{d}\right)$ is arbitrary (101) and (104) yield $\gamma \in \Gamma(\mu)$, which concludes the proof of Proposition B.1.

Appendix C. Density of the set of maps with prescribed boundary values.

Proposition C.1. Suppose that $d \geq 2, \Omega, \Lambda \subset \mathbf{R}^{d}$ are two open, bounded sets, that $\partial \Omega$ is Lipschitz, and that $\Lambda$ is convex. Let $\mathbf{u}, \mathbf{u}_{o} \in C^{1}(\Omega)^{d} \cap C(\bar{\Omega})^{d}$ be such that $\operatorname{det} D \mathbf{u}, \operatorname{det} D \mathbf{u}_{o}$ are positive, of class $C^{1}(\Omega)$, with $\operatorname{det} D \mathbf{u}+\frac{1}{\operatorname{det} D \mathbf{u}}$ and $\operatorname{det} D \mathbf{u}_{o}+$ $\frac{1}{\operatorname{det} D \mathbf{u}_{o}}$ in $L^{\infty}(\Omega)$. Suppose furthermore that $\mathbf{u}_{o}$ is one-to-one on $\Omega$, that $\mathbf{u}$ is oneto-one on $\Omega$, and that $\mathbf{u}(\Omega)=\mathbf{u}_{o}(\Omega)=\Lambda$. Then there exists a sequence $\left(\mathbf{u}_{n}\right) \subset$ $C^{1}(\Omega)^{d} \cap C(\bar{\Omega})^{d}$ of one-to-one maps from $\bar{\Omega}$ onto $\bar{\Lambda}$ converging almost everywhere in $\Omega$ to $\mathbf{u}$ and such that for each integer $n$

$$
\left\{\begin{array}{ccccc}
\operatorname{det} D \mathbf{u}_{n} & = & \operatorname{det} D \mathbf{u} & \text { almost everywhere in } & \Omega, \\
\mathbf{u}_{n} & = & \mathbf{u}_{o} & \text { on } & \partial \Omega .
\end{array}\right.
$$

Proof. Step 1. Using Theorem 7 in [9] we find $\mathbf{b} \in \operatorname{Diff}^{1}(\Omega) \cap \operatorname{Diff}^{0}(\bar{\Omega})$ such that

$$
\left\{\begin{array}{ccccc}
\operatorname{det} D \mathbf{u}_{0}(\mathbf{b}(\mathbf{x})) \operatorname{det} D \mathbf{b}(\mathbf{x}) & = & \operatorname{det} D \mathbf{u}(\mathbf{x}) & \text { in } & \Omega \\
\mathbf{b}(\mathbf{x}) & = & \mathbf{x} & \text { on } & \partial \Omega
\end{array}\right.
$$

Define the maps

$$
\mathbf{v}:=\mathbf{u}_{o} \circ \mathbf{b}, \quad \mathbf{s}:=\mathbf{u} \circ \mathbf{v}^{-1} .
$$

Clearly

$$
\left\{\begin{array}{ccccc}
\operatorname{det} D \mathbf{v} & = & \operatorname{det} D \mathbf{u} & \text { in } \quad & \Omega \\
\mathbf{v}(\mathbf{x}) & = & \mathbf{u}_{o}(\mathbf{x}) & \text { on } & \partial \Omega
\end{array}\right.
$$

We have that

$$
\mathbf{s}(\Lambda)=\mathbf{u}\left(\mathbf{b}^{-1}\left[\mathbf{u}_{o}^{-1}(\Lambda)\right]\right)=\mathbf{u}(\Omega)=\Lambda
$$

and $\mathbf{s}$ is measure-preserving in the sense that

$$
\int_{\Lambda} G(\mathbf{s}(\mathbf{y})) d \mathbf{y}=\int_{\Lambda} G(\mathbf{x}) d \mathbf{x}
$$

for all $G \in C_{o}\left(\mathbf{R}^{d}\right)$.

Step 2 . Since $\Lambda$ is convex and bounded, there exists a map $T \in \operatorname{Diff}^{1}\left(\Lambda,(0,1)^{d}\right) \cap$ $\operatorname{Diff}^{0}\left(\bar{\Lambda},[0,1]^{d}\right)$. One can choose $T$, for instance, to be the optimal map that rearranges $\frac{\chi_{\Lambda}}{|\Lambda|} d \mathbf{x}$ onto $\chi_{[0,1] d} d \mathbf{x}$ in the Monge problem, where optimality is measured against the cost function $c(\mathbf{x}-\mathbf{y})=|\mathbf{x}-\mathbf{y}|^{2}$. Using $T$ we deduce that the following known result for $[0,1]^{d}$ (see, for instance, [2] and [30]) holds for any convex, bounded 
set $\Lambda$ : there exists a sequence $\left(\mathbf{s}_{n}\right) \subset C^{1}(\Lambda)^{d} \cap C(\bar{\Lambda})^{d}$ of maps from $\bar{\Lambda}$ onto $\bar{\Lambda}$ that are one-to-one on $\Lambda$, that converge pointwise almost everywhere in $\Lambda$ to s such that

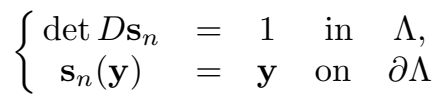

for $n=1,2, \ldots$. Define

$$
\mathbf{u}_{n}(\mathbf{x}):=\mathbf{s}_{n}(\mathbf{v}(\mathbf{x})) \quad(\mathbf{x} \in \bar{\Omega}) .
$$

By (107) and (108) we deduce that $\left(\mathbf{u}_{n}\right)$ satisfies the conclusions of Proposition C.1.

Appendix D. Background on the Monge problem. In this section we present a brief description of the Monge problem, a theory which has attracted a lot of attention. Throughout this section we keep our focus only on the case that is relevant to the study of solid crystals, the case studied by [3], [19], etc. Let $\mu=f d \mathbf{x}$, $\nu=g d \mathbf{x}$ be finite measures on $\mathbf{R}^{d}$ with equal total mass. Let $O_{1}, O_{2} \subset \mathbf{R}^{d}$ be two open sets such that $\bar{O}_{1}$ is the support of $\mu$ and $\bar{O}_{2}$ is the support of $\nu$. The Monge mass transport problem consists of finding an optimal way of rearranging $\mu$ onto $\nu$ against a cost function which we choose here to be $c(\mathbf{x}-\mathbf{y})=|\mathbf{x}-\mathbf{y}|^{2}$. The corresponding variational problem is to minimize the total work

$$
K[T]:=\int_{\mathbf{R}^{d}}|\mathbf{x}-T \mathbf{x}|^{2} d \mu(\mathbf{x})
$$

over the set $\mathcal{T}$ of all Borel maps $T: \mathbf{R}^{d} \rightarrow \mathbf{R}^{d}$ that push $\mu$ forward to $\nu$. Define

$$
K^{\prime}[S]:=\int_{\mathbf{R}^{d}}|\mathbf{y}-S \mathbf{y}|^{2} d \nu(\mathbf{y})
$$

and let $\mathcal{S}$ be the set of all Borel maps $S: \mathbf{R}^{d} \rightarrow \mathbf{R}^{d}$ that push $\nu$ forward to $\mu$. The following results are known in a setting more general than the one herein.

Proposition D.1 (general theorem).

(i) Existence and uniqueness of optimal maps: there exists a unique $T_{o}$ minimizing $K$ over $\mathcal{T}$. Likewise, there exists a unique $S_{o}$ minimizing $K^{\prime}$ over $\mathcal{S}$. We have that $S_{o}\left(T_{o}(\mathbf{x})\right)=\mathbf{x}$ for $\mu$-almost every $\mathbf{x} \in \mathbf{R}^{d}, T_{o}\left(S_{o}(\mathbf{y})\right)=\mathbf{y}$ for $\nu$-almost every $\mathbf{y} \in \mathbf{R}^{d}$.

(ii) Characterization of optimal maps: a map $T_{o}$ is a minimizer of $K$ over $\mathcal{T}$ if and only if $T_{o} \in \mathcal{T}$ and $T_{o}$ is the gradient of a convex function $\psi_{o}: \mathbf{R}^{d} \rightarrow \mathbf{R} \cup\{+\infty\}$. Similarly, a map $S_{o}$ is a minimizer of $K^{\prime}$ over $\mathcal{S}$ if and only if $S_{o} \in \mathcal{S}$ and $S_{o}$ is the gradient of a convex function $\phi_{o}: \mathbf{R}^{d} \rightarrow \mathbf{R} \cup\{+\infty\}$.

(iii) The sets $T_{o}\left(O_{1}\right)$ and $O_{2}$ coincide up to a set of zero measure.

Proof. We refer the reader to [19].

Proposition D.2 (smoothness of optimal maps). Assume that $O_{1}, O_{2}$ are bounded, $\left|\partial O_{1}\right|=\left|\partial O_{2}\right|=0, f+1 / f \in L^{\infty}\left(O_{1}\right), g+1 / g \in L^{\infty}\left(O_{2}\right), O_{2}$ is convex, and $\psi_{o}, \phi_{o}$ are the convex functions obtained in Proposition D.1. Then we have the following:

(i) $\psi_{o} \in C^{1, s}\left(O_{1}\right)$ for some $0<s<1$, and $\psi_{o}$ is strictly convex in $O_{1}$.

(ii) If in addition $O_{1}$ is convex, then $\psi_{o} \in C^{1, s}\left(\bar{O}_{1}\right)^{d}$ for some $0<s<1$.

(iii) If $O_{1}$ is convex and in addition $f \in C^{0, \bar{s}}\left(O_{1}\right), g \in C^{0, \bar{s}}\left(O_{2}\right)$, then $D \psi_{o} \in$ $C^{1, s}\left(O_{1}\right)^{d} \cap C^{0, \bar{s}}\left(\bar{O}_{1}\right)^{d}, D \phi_{o} \in C^{1, s}\left(O_{2}\right)^{d} \cap C^{0, \bar{s}}\left(\bar{O}_{2}\right)^{d}$ for all $0<s<\bar{s}$. We have that $D \psi_{o} \in \operatorname{Diff}^{0}\left(\bar{O}_{1}, \bar{O}_{2}\right)$. 
Proof. Smoothness properties of $\psi_{o}$ and $\phi_{o}$ as stated in (i), (ii), and (iii) are established in [4], [5], and [6]. If $D \psi_{o} \in C^{0, \bar{s}}\left(\bar{O}_{1}\right)^{d}$ and $D \phi_{o} \in C^{0, \bar{s}}\left(\bar{O}_{2}\right)^{d}$, then by Proposition D.1 we have that $D \psi_{o} \in \operatorname{Diff}^{0}\left(\bar{O}_{1}, \bar{O}_{2}\right)$.

Acknowledgments. The authors would like to thank B. Dacorogna, D. Kinderlehrer, and A. Swiech for comments on the paper.

\section{REFERENCES}

[1] G. Alberti, On the structure of singular sets of convex functions, Calc. Var. Partial Differential Equations, 2 (1994), pp. 17-27.

[2] Y. BREniER, personal notes.

[3] Y. Brenier, Décomposition polaire et réarrangement monotone des champs de vecteurs, C.R. Acad. Sci. Paris Sér. I Math., 305 (1987), pp. 805-808.

[4] L.A. Caffarelli, The regularity of mappings with a convex potential, J. Amer. Math. Soc., 5 (1992), pp. 99-104,.

[5] L. CAffarelli, Boundary regularity of maps with convex potentials, Comm. Pure Appl. Math., 45 (1992), pp. 1141-1151.

[6] L.A. Caffarelli, Boundary regularity of maps with convex potentials. II, Ann. of Math. (2), 144 (1996), pp. 453-496.

[7] M. Chipot and D. Kinderlehrer, Equilibrium configurations of crystals, Arch. Rational Mech. Anal. , 103 (1988), pp. 237-277.

[8] B. Dacorogna, Direct Methods in the Calculus of Variations, Springer-Verlag, Berlin, 1989.

[9] B. Dacorogna AND J. Moser, On a partial differential equation involving the Jacobian determinant, Ann. Inst. H. Poincare Anal. Non Linéaire, 7 (1990), pp. 1-26.

[10] I. Ekeland And R. Teman, Convex Analysis and Variational Problems, Stud. Math. Appl. 1, North-Holland, Amsterdam, 1976.

[11] J.L. Ericksen, Special topics in electrostatics, Advance in Applied Math. Mech., 17 (1977), pp. $188-224$.

[12] J.L. ERICKSen, Twinning of crystals, in Metastability and Incompletely Posed Problems, S. Antman, J.L. Ericksen, D. Kinderlehrer, and I. Müller, eds., Springer-Verlag, Berlin, 1987, pp. 77-94.

[13] L.C. Evans and R.F. Gariepy, Measure Theory and Fine Properties of Functions, Stud. Adv. Math., CRC Press, Boca Raton, FL, 1992.

[14] I. FonsecA, Variational methods for elastic crystals, Arch. Rational Mech. Anal., 97 (1987), pp. 189-220.

[15] I. FONSECA, The lower quasiconvex envelope of the stored energy function for an elastic crystals, J. Math. Pures Appl. (9), 67 (1988), pp. 175-195.

[16] I. Fonseca And W. Gangbo, Degree Theory in Analyis and Applications, Clarendon Press, Oxford, UK, 1995.

[17] I. Fonseca and L. Tartar, The displacement problem for elastic crystals, Proc. Royal Soc. Edinburgh Sect. A, 113 (1989), pp. 159-180.

[18] W. Gangbo, An elementary proof of the polar factorization of vector-valued functions, Arch. Rational Mech. Anal., 128 (1994), pp. 381-399.

[19] W. Gangbo and R.J. McCann, The geometry of optimal transportation, Acta Math., 177 (1996), pp. 113-161.

[20] R.D. James, Mechanics of Coherent Phase Transformations in Solids, MRL Report, Brown University, Division of Engineering, 1982, pp. 185-211.

[21] R.D. James, The arrangement of coherent phases in a loaded body, in Phase Transformations and Material Instabilities in Solids, M. Gurtin, ed., Academic Press, New York, 1984, pp. 79-98.

[22] R.D. James, Stress free joints and polycrystals, Arch. Rational Mech. Anal., 86 (1984), pp. 1337.

[23] R.D. James, Displacive phase transformations in solids, J. Mech. Phys. Solids, 34 (1986), pp. 359-394.

[24] D. KINDERLEhrer, Twinning of crystals. II, in Metastability and Incompletely Posed Problems, S. Antman, J.L. Ericksen, D. Kinderlehrer, and I. Müller, eds., Springer-Verlag, Berlin, 1987, pp. 185-211.

[25] G. PARRY, One phase transitions involving internal strain, Internat. J. Solids Structures, 17 (1981), pp. 361-378. 
[26] M. PitTeri, Reconciliation of local and global symmetries of crystals, J. Elasticity, 14 (1984), pp. $175-190$.

[27] M. PItTeRI, On the kinematics of mechanical twinning in crystals, Arch. Rational Mech. Anal., 88 (1985), pp. 25-57.

[28] M. Pitteri, On type-2 twins in crystals, Internat. J. Plasticity, 2 (1986), pp. 99-106.

[29] M. Pitteri, A contribution to the description of natural states for elastic crystalline solids, in Metastability and Incompletely Posed Problems, IMA Vol. Math. Appl. 3, S. Antman, J.L.

Ericksen, D. Kinderlehrer, and I. Müller, eds., Springer-Verlag, Berlin, 1987, pp. 295-310.

[30] M. Roesch, Thèse de Doctorat, Université Pierre et Marie Curie, Paris VI, 1995. 